\title{
THE INTRODUCTION OF IDEAL ELEMENTS AND A NEW
}

\section{DEFINITION OF PROJECTIVE $n$-SPACE*}

\author{
BY \\ FREDERICK WILLIAM OWENS
}

Introduction.

The ideal elements of projective geometry are usually introduced by means of the parallel and congruence axioms. The idea of defining the ideal elements without the assumption of the parallel axiom is due to KLEIN. $\dagger$ It was developed by Pasch; $\ddagger$ by Schur; $§$ by Bonola; $\|$ and by Veblen. 9 In all of these developments a three-dimensional gecmetry is assumed. The problem of defining the ideal elements in a plane geometry satisfying only order relations is closely connected with the problem of finding the necessary and sufficient condition that a plane may be a part of a three-space in which the axioms of order are satisfied. This condition is stated by HILBER' ${ }^{*} *$ to be the validity of the Desargues theorem in the plane. The Desargues theorem may be proved in a three-dimensional space satisfying only order relations, but can not be proved in the corresponding plane geometry, without additional assumptions, e. g., of the parallel and congruence axioms.

In this paper plane axioms of order will be assumed in the form given them by VEbLen, the undefined elements being taken as the point, and a relation among points, called order. The first eight axioms are identical, except for notation, with his. Another axiom, one of closure, is then introduced, limiting the set of points considered to a plane. Two more axioms are then introduced, forms of the Desargues theorem, and of its converse, in terms of the set of points satisfying only order relations.

\footnotetext{
* Presented to the Society (Chicago) under a different title, A pril 22, 1905.

$\uparrow$ Ueber die sogencennte Nicht-Euklidische Geometrie, Mathematische Annalen, vol. 6 (1872), p. 132.

$\ddagger$ Vorlesungen über neucic Geometrie, pp. 40-72.

$\S$ Ueber die Einfïhrung der sogencunten idealen Elcmente in die projectire Geometrie, $\mathbf{M}$ athematische Annalen, vol. 39 (1891), pp. 113-128.

$\|$ Sulla introduziome degle enti improprii in Geometria projettiva, Giornale di Matematiche, vol. 38 (1900), p. 105.

IA system of arioms for geometry, these Transactions, vol. 5 (1904), pp. 343-384.

* * Grundlugen der Cieometrie, Festschrift (1899), § 30.
} 
On the basis of these eleven axioms, a new set of elements, called pencils, including in particular the pencils of lines centered at points, are defined, and their properties deduced. This set of pencils is shown to satisfy the incidence axioms and the general Desargues theorem of projective geometry, but not necessarily the Pascal theorem, well known * to be independent of the Desargues theorem, and even stronger assumptions. It is also shown that if we leave out of this set of pencils a subset called a range, the remaining elements satisfy the original axioms, I to XI.

In Section IV, a new set of elements called three-points, including in particular the projective points (i. e. pencils) of the plane, is defined, and this set is shown to form a projective three-space containing the plane set as a subset.

In Section $\mathrm{V}$, an extension is made to $n$-space by generalizing the method of Section IV.

The notation used, though sometimes cumbersome, is very convenient in giving a concise expression for the proofs ; and by displaying the configurational character of many of the figures used, it has been found of value in devising the proofs of the theorems. The points which are characterized by Axioms I-XI will be denoted by the symbol $P_{k}$, the $P$ simply indicating that the element belongs to the class of points, and the subscript being a mark, not necessarily a single digit or letter, and the order of the digits or letters in the subscript immaterial, used to distinguish the various points ; for instance, conveniently, the notation $P_{x y}, x, y=1, \cdots, 5, x \neq y$, and $P_{x y}=P_{y x}$, will be used for the configuration of ten points called the Desargues configuration; the notation $l_{k}$ will be used for lines, the $k$ again being a mark of one or more digits or letters, the order of which is immaterial ; the notation $l_{123}$ will be used where convenient for a line containing points $P_{12}, P_{13}, P_{23}$. Such implications of the notation, while useful, are avoided in the statement of the proofs, as far as possible, but the notation is used consistently, even the axioms themselves being stated in it, in order to accustom the reader to it. Other forms of notation will be explained, as they occur.

The independence of the Axioms $\mathrm{X}$ and XI from Axioms I-IX may be shown from the Non-Desarguesian geometry of Moulton $\dagger$ if the "line of break" of this geometry is a line of our points. The relation of Axioms $\mathrm{X}$ and XI on the basis of Axioms I-IX has not been determined.

This problem was first suggested to the author by Professor O. Veblen, to whom, and to Professor E. H. Moone, he tenders his grateful acknowledgment for many helpful suggestions and criticisms.

* Grundlagen der Geometrie, Festschrift, §31. The form of Pascal's theorem here referred to is, of course, that for the degenerate conic. (Theorem of Pappus.)

$\dagger$ A simple Non-Desarguesian geometry, Transactions of tbe A merican Mathematical Society, vol. 3 (1902), pp. 192-195. 


\section{The Axioms and Definitions.}

Axiom I. There exist at least two distinct points.

Axıom II. If points $P_{1}, P_{2}, P_{3}$ are in the order $P_{1} P_{2} P_{3}$, they are in the order $P_{3} P_{2} P_{1}$.

Axıom III. If points $P_{1}, P_{2}, P_{3}$ are in the order $P_{1} P_{2} P_{3}$, they are not in the order $P_{2} P_{3} P_{1}$.

Axıом IV. If points $P_{1}, P_{2}, P_{3}$ are in the order $P_{1} P_{2} P_{3}$, then $P_{1}$ is distinct from $P_{3}$.

Axiom V. If $P_{1}$ and $P_{2}$ are any two distinct points, there exists a. point $P_{3}$, such that $P_{1}, P_{2}, P_{3}$ are in the order $P_{1} P_{2} P_{3}$.

If $P_{1}$ and $P_{2}$ stand for the same points, we write $P_{1}=P_{2}$; if for distinct points, $P_{1} \neq P_{2}$.

Definition 1. The line $P_{1} P_{2}$, or conveniently, $l_{12}$, consists of $P_{1}$ and $P_{2}$ and all points $P_{k}$ in any of the orders, $P_{1} P_{k} P_{2}, P_{1} P_{2} P_{k}, P_{k} P_{1} P_{2}$. The subscripts 1,2 in the notation $l_{12}$ refer to the points by which the line is determined.

The points $P_{k}$ in the order $P_{1} P_{k} P_{2}$ constitute the segment $P_{1} P_{2}$.

Axıом VI. If points $P_{3}$ and $P_{4}\left(P_{3} \neq P_{4}\right)$ lie on the line $l_{12}\left(\right.$ i. e., $\left.P_{1} P_{2}\right)$ then $P_{1}$ lies on the line $l_{34}$ (i. e., $P_{3} P_{4}$ ).

Axıom VII. If there exist three distinct points, there exist three points $P_{1}, P_{2}, P_{3}$ not in any one of the orders $P_{1} P_{2} P_{3}, P_{1} P_{3} P_{2}$, or $P_{3} P_{1} P_{2}$.

Definition 2. Points of the same line are collinear. Three distinct noncollinear points, $P_{1}, P_{2}, P_{3}$ are the vertices of a triangle $P_{1} P_{2} P_{3}=T_{123}$, whose sides are the segments $P_{1} P_{2}, P_{1} P_{3}, P_{2} P_{3}$, and whose boundary consists of its vertices and the points on its sides. Two triangles having the same vertices are identical.

Axıом VIII. (Triangle transversal axiom.) If three distinct points $P_{1} P_{2} P_{3}$ do not lie on the same line, and $P_{4}$ and $P_{5}$ are two points in the orders $P_{2} P_{3} P_{4}$ and $P_{3} P_{5} P_{1}$, respectively, then a point $P_{6}$ exists in the order $P_{1} P_{6} P_{2}$, and such that $P_{4}, P_{5}, P_{6}$ lie on the same line.

Definition 3. If $P_{1}, P_{2}, P_{3}$ are three non-collinear points, the plane $P_{1} P_{2} P_{3}$ consists of all the points collinear with any two points of the sides of the triangle $P_{1} P_{2} P_{3}^{\prime}$.

Axıom IX. The set of points $P_{k}$ here considered constitutes a plane.

Definition 4. A triangle is said to lie on three lines if it has a vertex on each of these lines.

Definition 5. Three distinct lines $l_{340}, l_{341}, l_{342}$ are in pencil if there exists on these lines a pair of triangles having no side common $P_{30} P_{31} P_{32}$ and $P_{40} P_{41} P_{42}, P_{30}$ and $P_{40}, P_{30} \neq P_{40}$, being points of the line $l_{340}$, etc., such that the lines $P_{30} P_{31}$ and $P_{40} P_{41}$ meet* in a point $P_{01}$, the lines $P_{31} P_{32}$ and

* Two lines meet only if distinct. 
$P_{41} P_{42}$ meet in a point $P_{12}$, and the lines $P_{30} P_{32}$ and $P_{40} P_{42}$ meet in a point $P_{02}$, and such that the points $P_{01}, P_{02}, P_{12}$ are collinear.

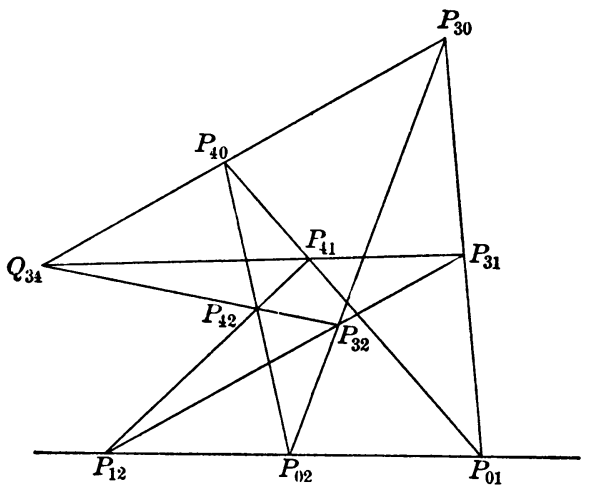

Fig. 1.

The figure of Definition 5 (see Fig. 1) will be called a Desargues configuration, and the notation

$$
\begin{array}{lll}
P_{12} & P_{02} & P_{01} \\
\hdashline P_{40} & P_{41} & P_{42} \\
P_{30} & P_{31} & P_{32} \\
& Q_{34} &
\end{array}
$$

will be used when by hypothesis the triads of points, $P_{12} P_{02} P_{01}, P_{12} P_{41} P_{42}$, $P_{12} P_{31} P_{32}, P_{02} P_{40} P_{42}, P_{02} P_{30} P_{32}$, $P_{01} P_{40} P_{41}, P_{01} P_{30} P_{31}$ are collinear, and the triads $P_{30} P_{31} P_{32}$ and $P_{40} P_{41} P_{42}$ are vertices of triangles; by the above definition, then, the lines $P_{40} P_{39}, P_{41} P_{31}$, and $P_{42} P_{32}$ will be in pencil, $Q_{34}$.

Definition 6. The set of all lines in pencil with a given pair, together with this pair, constitute a pencil, determined by the two lines, and by Theorem VI, determinable by any two lines in it. The notation $Q_{k}$ will be used for pencils, the mark $k$, as before, being any convenient symbol of one, two, or more digits or letters, these usually referring to the lines by which it is determined, and the order of the digits of the subscript being immaterial as far as denoting the pencil is concerned. A line is said to belong to a pencil if it is one of the lines of the pencil.

Axıom X. If $l_{340}, l_{341}, l_{342}$ are three lines in a pencil, and $P_{30} P_{31} P_{32}$ and $P_{40} P_{41} P_{42}$ are any two triangles on these lines, $P_{30}$ and $P_{40}$ lying on $l_{340}$ (but not necessarily distinct), etc., such that the lines

$$
\begin{array}{lll}
P_{30} P_{31}=l_{301} & \text { and } & P_{40} P_{41}=l_{401} \text { meet in a point } P_{01}, \\
P_{31} P_{32}=l_{312} & \text { and } & P_{41} P_{42}=l_{412} \text { meet in a point } P_{12}, \\
P_{30} P_{32}=l_{302} & \text { and } & P_{40} P_{42}=l_{402} \\
\text { meet in a point } P_{02},
\end{array}
$$

and $P_{01}, P_{12}, P_{02}$ are distinct and determinate, then $P_{01}, P_{12}$, and $P_{02}$ are collinear.

The figure of Axiom $\mathrm{X}$ (see Fig. 2) will be represented by the notation

$$
\begin{array}{lll} 
& Q_{34} \\
P_{30} & P_{31} & P_{32} \\
P_{40} & P_{41} & P_{42} \\
\hline P_{12} & P_{01} & P_{01}
\end{array}
$$


which indicates that by hypothesis the lines $P_{30} P_{40}, P_{31} P_{41}, P_{32} P_{42}$ are in pencil $Q_{34}$, and the triads of points $P_{30}$ $P_{31} P_{32}$ and $P_{40} P_{41} P_{42}$ are the vertices of triangles on these lines, and the triads of points $P_{30} P_{31} P_{01}, P_{40} P_{41} P_{01}$, $P_{30} P_{32} P_{02}, \quad P_{40} P_{42} P_{02}, \quad P_{31} P_{32} P_{12}$, $P_{41} P_{42} P_{12}$, exist and are respectively collinear. Axiom $\mathrm{X}$ states, then, that the points $P_{12} P_{02} P_{01}$ are collinear.

Definitron 7. Three distinct pencils $Q_{01}, Q_{12}, Q_{02}$ are in range, if there exist two triangles, $P_{30} P_{31} P_{32}$ and $P_{40} P_{41} P_{42}$, whose sides are all distinct and such that $P_{30} P_{31}$ and $P_{40} P_{41}$ belong to $Q_{01}, P_{31} P_{32}$

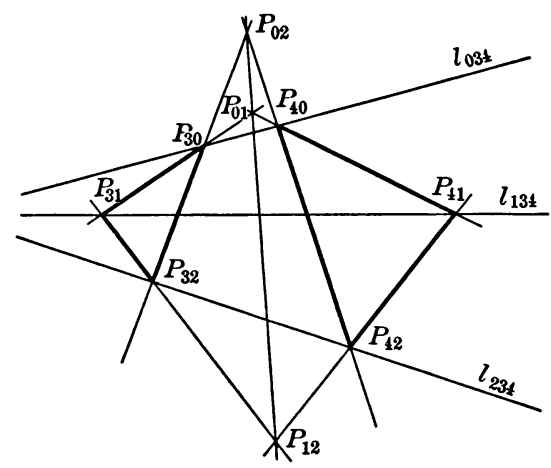

Fig. 2. and $P_{41} P_{42}$ belong to $Q_{12}$, and $P_{30} P_{32}$ and $P_{40} P_{42}$ belong to $Q_{02}$; and such that the lines $P_{30} P_{40}, P_{31} P_{41}$, and $P_{32} P_{42}$ are in a pencil $Q_{34}$.

The notation used for the figure of Definition 7 will be identical with that of Axiom $\mathrm{X}$, except that for $P_{12}, P_{02}, P_{01}$ corresponding symbols $Q_{12}, Q_{02}, Q_{01}$ will be used, thus :

$$
\begin{array}{ccc} 
& Q_{34} \\
P_{30} & P_{31} & P_{32} \\
P_{40} & P_{41} & P_{42} \\
\hline Q_{12} & Q_{20} & Q_{01}
\end{array}
$$

It will be observed that whenever the hypothesis of Axiom $\mathrm{X}$ is fulfilled, so is the hypothesis in Definition 7, and to the points $P_{12} P_{02} P_{01}$ of Axiom X correspond pencils $Q_{12} Q_{02} Q_{01}$ which by Definition 7 are in range; for whether the pairs of lines $P_{30} P_{32}$ and $P_{40} P_{42}, P_{31} P_{32}$ and $P_{41} P_{42}$, and $P_{30} P_{31}$ and $P_{40} P_{41}$, determine points or not, they do determine pencils (cf. Corollary to Theorem VII).

Axıom XI. If three pencils $Q_{01}, Q_{02}, Q_{12}$ are in range, every pair of triangles $P_{30} P_{31} P_{32}$ and $P_{40} P_{41} P_{42}$, such that

lines $P_{30} P_{31}$ and $P_{40} P_{41}$ belong to $Q_{01}$,

lines $P_{31} P_{32}$ and $P_{41} P_{42}$ belong to $Q_{12}$,

lines $P_{30} P_{32}$ and $P_{40} P_{42}$ belong to $Q_{02}$,

has the property that the lines $P_{30} P_{40}, P_{31} P_{41}$ and $P_{32} P_{42}$ are in a pencil $Q_{34}$.

The figure of Axiom XI will be denoted by the notation:

\begin{tabular}{ccc}
$Q_{12}$ & $Q_{20}$ & $Q_{01}$ \\
\hline$P_{30}$ & $P_{31}$ & $P_{32}$ \\
$P_{40}$ & $P_{41}$ & $P_{42}$ \\
& $Q_{34}$ &
\end{tabular}


The inversion of the notational form is to call attention to the change in the hypothesis. That Definitions 6 and 7 and Axioms $X$ and $X I$ all involve the Desargues configuration explains the similarity of the notation.

Definition 8. The set of all pencils in range with a given pair of ranges, together with this pair, constitutes a range. The notation $r_{k}$ will be used for ranges, the mark $k$ being again any convenient digit or letter or combination of them in which the order is immaterial, so far as denoting the range is concerned.

From Axioms I-IX, such theorems as the following may be deduced (cf. VEBLEN, loc. cit.):

a) Between any two distinct points of a line there is another point $\left(P_{1}\right.$ is between $P_{2}$ and $P_{3}$ if they are in the order $\left.P_{2} P_{1} P_{3}\right)$.

b) If a line, not a side of a triangle, meets the perimeter of the triangle in one point not a vertex, then it meets the perimeter in another distinct point.

c) To any four points of a line the notation $P_{1}, P_{2}, P_{3}, P_{4}$, can be so assigned that they are in the order, $P_{1} P_{2} P_{3} P_{4}$, i. e. in the orders, $P_{1} P_{2} P_{3}$, $P_{1} P_{3} P_{4}, P_{1} P_{2} P_{4}$, and $P_{2} P_{3} P_{4}$.

d) Any line divides the plane into two regions which may be denoted by + and - , and have the property that any segment joining a point of + to a point - contains a point of the line.

e) Two non-intersecting lines divide a plane into three regions, which can be conveniently denoted by,,+++--- .

$f$ ) Two intersecting lines divide a plane into four regions, which may be denoted by,,,+++--+-- .

g) Three lines forming an actual triangle divide the plane into seven regions, which may be denoted by,,,,,,+++++-+-++----+-++-+- .

\section{Section I.}

\section{Consequences of Axioms $I-X$.}

Our main object in Section $I$ is to show that a line is uniquely determined by a point and the fact that it is in pencil with two other given lines neither of which passes through the point. This is done without any use of Axiom XI.

ThEOREM I. If the lines $l_{034}, l_{134}, l_{234}$ are in pencil $Q_{34}$, and $l_{034}$ and $l_{134}$ meet in a point $P_{30}$, then $l_{234}$ passes through $P_{30}$. (Fig. 3.)

Proof. Suppose the theorem untrue. Take $P_{32}$, any point of $l_{234}\left(P_{32}\right.$ not a point of $l_{034}$ or $l_{134}$ ). Take on $l_{134}$ points $P_{71}$ and $P_{61}$ each distinct from $P_{30}$, not on $l_{234}$, in the order $P_{71} P_{30} P_{61}$, and such there is no point of $l_{234}$ on the segment $P_{61} P_{71}$. The line $l_{034}$ cuts the side $P_{71} P_{61}$ of the triangle $P_{71} P_{61} P_{32}$. Hence it cuts either the segment $P_{32} P_{71}$ or the segment $P_{32} P_{61}$ in a point $P_{t}$. Call the side cut $P_{32} P_{31}$, so that either $P_{71}$ or $P_{61}$ is $P_{31}$, and call the other $P_{81}$. Choose a point $P_{02}$ in the order $P_{32} P_{30} P_{02}$, and a point $P_{y}$ in the order 
$P_{31} P_{32} P_{y}$. The segment $P_{02} P_{y}$ contains a point of the segment $P_{32} P_{81}$ or does not. If it does, use $P_{y}$ as $P_{01}$ below. If not, take a point $P_{r}$ on the segment $P_{32} P_{81}$, and the line $P_{02} P_{r}$ will meet the segment $P_{32} P_{y}$ in a point

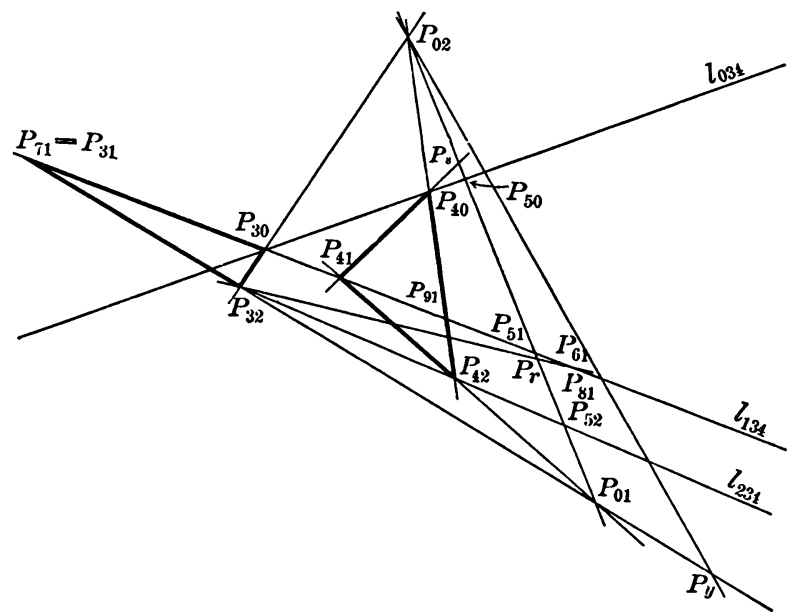

FIG. 3.

$P_{01}$, which is in the order $P_{02} P_{r} P_{01}$. Then the segment $P_{02} P_{01}$ will meet $l_{034}$, $l_{134}$, and $l_{234}$ in points $P_{50}, P_{51}, P_{52}$, respectively, in the order $P_{02} P_{50} P_{51} P_{r} P_{52} P_{01}$. Take $P_{41}$ in the order $P_{30} P_{41} P_{51}$. Then the segment $P_{41} P_{01}$ meets $l_{234}$ in a point $P_{42}$ of the segment $P_{32} P_{52}$. The segment $P_{42} P_{02}$ contains points, $P_{40}$ on the line $l_{034}$, and $P_{91}$ on the line $l_{134}$, in the order $P_{02} P_{40} P_{91} P_{42}$, and $P_{91}$ is in the order $P_{30} P_{41} P_{91} P_{51}$. Therefore lines $P_{41} P_{41)}$ and $P_{02} P_{01}$ determine a point $P_{0}$ in the orders $P_{41} P_{40} P_{\text {, and }} P_{02} P_{6} P_{50}$. We have then the Desargues configuration

\begin{tabular}{ccc} 
& $Q_{34}$ \\
$P_{30}$ & $P_{31}$ & $P_{32}$ \\
$P_{40}$ & $P_{41}$ & $P_{42}$ \\
\hline$P_{01}$ & $P_{02}$ & $P_{41}$,
\end{tabular}

in which the hypothesis of Axiom $\mathrm{X}$ is satisfied, but $P_{01}, P_{02}, P_{41}$ are not collinear. Hence the assumption that $l_{234}$ does not pass through $P_{02}$ is contradictory to Axiom X. Hence the theorem is true.

Theorem II. Any three lines $l_{034}, l_{134}, l_{234}$ incident at a point $P_{34}$ are in a pencil $Q_{34}$. (Fig. 4.)

Proof. Choose $P_{30} \neq P_{34}$ on $l_{034}$ and $P_{61} \neq P_{34}$ on $l_{134}$, and $P_{32} \neq P_{34}$ on $l_{234}$, in the order $P_{32} P_{61} P_{30}$. Then choose $P_{02}$ in the order $P_{32} P_{61} P_{30}^{\prime} P_{02}$; $P_{31}$ in order $P_{34} P_{61} P_{31} ;$ and $P_{12}$ in the order $P_{31} P_{32} P_{12}$, and such that segment $P_{32} P_{12}$ contains no point of line $P_{34} P_{02}$. Then, by consideration of 
triangle $P_{02} P_{32} P_{12}, P_{02} P_{12}$ contains points $P_{50}, P_{51}$, in order $P_{02} P_{50} P_{51} P_{12}$,

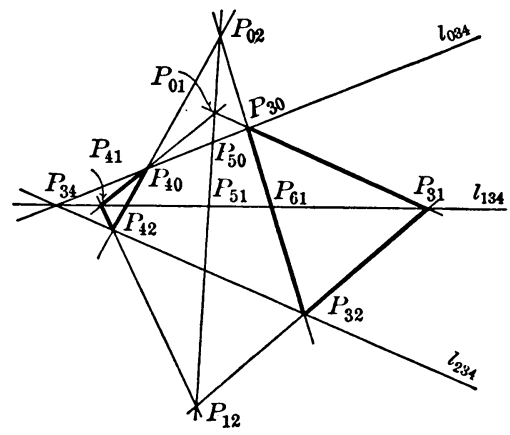

FIG. 4. and $P_{50}$ is on the segment $P_{34} P_{30}$, and $P_{51}$ is on the segment $P_{34} P_{61} \cdot P_{31} P_{30}$ meets $P_{02} P_{12}$ in a point $P_{01}$, in the order $P_{02} P_{01} P_{50}$. Take $P_{41}$ on the segment $P_{34} P_{51}$. The segment $P_{41} P_{01}$ contains $P_{40}$ on the segment $P_{34} P_{59} \cdot P_{02} P_{40}$ meets $P_{41} P_{12}$ in a point $P_{42}$. Then $P_{32} P_{42}$ is a line in pencil with $l_{034}$ and $l_{134}$. By Theorem I, the line $P_{32} P_{42}$ must pass through $P_{34}$, but this line contains the points $P_{32}$ and $P_{34}$, and hence is the line $l_{234}$.

Corollary. If three lines are in pencil

they meet in a point, or else no two of them have a point in common.

Definition 8. A line $l_{k}$ is between the non-intersecting lines $l_{m}$ and $l_{n}$, if every segment $P_{m} P_{n}\left(P_{m}\right.$ on $l_{m}, P_{n}$ on $\left.l_{n}\right)$ contains a point $P_{k}$ of $l_{k}$.

Definition 9. If three lines are in pencil and no two of them intersect, we will call the pencil non-intersecting.

TheOREM III. If $l_{034}, l_{134}, l_{234}$ are three lines of a non-intersecting pencil, $Q_{34}$, then one of them is always between the other two.

Lемma. If $l_{k}, l_{m}, l_{n}$ are three non-intersecting lines, and if $l_{m}$ cuts one segment joining points of $l_{k}$ and $l_{n}$, then it cuts every such segment, and hence is between $l_{k}$ and $l_{n}$. The proof follows readily from Axiom VIII and is omitted.

Proof of Theorem III. (Fig. 5.) Take $P_{30}$ on $l_{034}, P_{31}$ on $l_{134}$, and $P_{32}$ on $l_{234}$. Then not more than one of the lines $l_{034}, l_{134}, l_{234}$ enters the triangle $P_{30} P_{31} P_{32}$, since by hypothesis the pencil is non-intersecting. Then, if one does, it cuts the opposite side of the triangle and hence by the lemma it is between the other two lines. We will now prove that the case of all three of the lines $l_{034}, l_{134}, l_{234}$ exterior to the triangle can not occur. Sup-

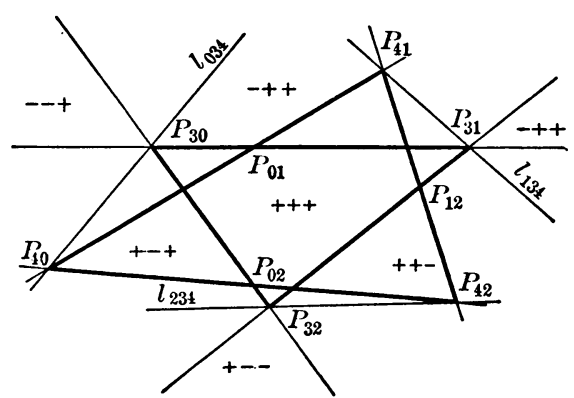

Fig. 5. pose that it did occur. Let the plane be divided by the sides of the triangle into seven regions as in Theorem $g$, p. 146 .

Choose $P_{40}$ on $l_{034}$ in +-+ , and $P_{41}$ on $l_{134}$ in -++ , and $P_{42}$ on $l_{234}$ in ++ . Then $P_{40} P_{41}$ meets segment $P_{30} P_{31}$ in a point $P_{01}$, and $P_{41} P_{42}$ meets segment $P_{31} P_{32}$ in a point $P_{12}$, and $P_{42} P_{40}$ meets segment $P_{32} P_{30}$ in a point $P_{02}$ and by hypothesis we have (2); but the points $P_{12}, P_{02}, P_{01}$ are not collinear since they are on distinct sides of the triangle. Hence our supposition that no one of the lines enters the triangle is impossible. 
Definition 10. If three lines of a pencil are incident at a point, then their common point is the center of the pencil.

TheOREM IV. If $l_{034}, l_{134}$, and $l_{234}$ are three lines in pencil, and such that, if the pencil be non-intersecting, ${ }^{*} l_{134}$ is between $l_{034}$ and $l_{234}$, and $l_{934}\left(l_{934} \neq l_{234}\right)$ is a line meeting $l_{234}$ in a point $P_{32}\left(P_{32}\right.$ not on $l_{034}$ and not on $\left.l_{134}\right)$, then $l_{034}$, $l_{134}$, and $l_{934}$ are not in pencil.

Proof. (A) If the pencil has a center, the theorem follows from Theorem I.

$(B)$ Suppose the pencil has no center. (Fig. 6.)

Suppose there were a line $l_{93 t}$ satisfying the conditions of the hypothesis. Then $l_{934}$ can not meet either $l_{034}$ or $l_{134}$, by Theorem I, and also $l_{134}$ will be between $l_{034}$ and $l_{934}$. Let $P_{80}$ and $P_{70}$ be any two distinct points on $l_{034}$. Let $P_{12}$ be any point in the order $P_{80} P_{32} P_{12}$. The segment $P_{80} P_{32}$ meets $l_{134}$ in a point $P_{31}$. Since neither $l_{234}$ nor $l_{934}$ meets either $l_{034}$ or $l_{134}$, the line $P_{70} P_{12}$ meets $l_{134}$ in a point $P_{41}, l_{234}$ in a point $P_{42}$, and $l_{934}$ in a point $P_{49}$, and these

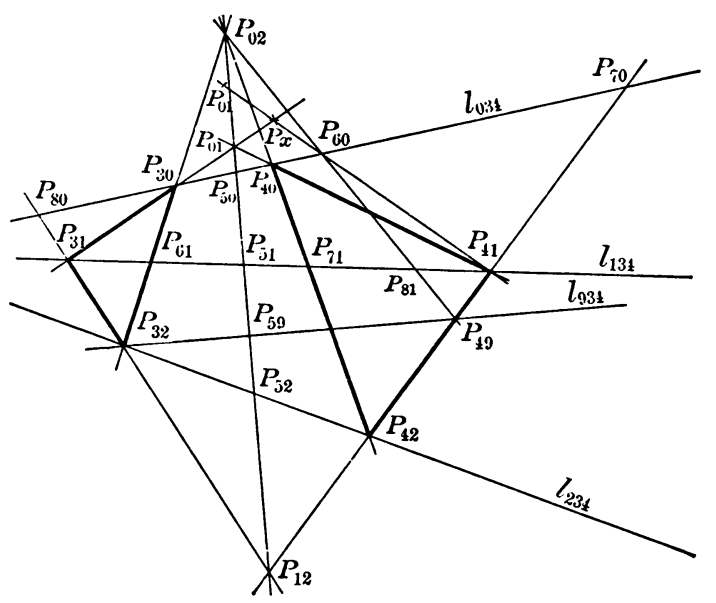

Fig. 6. points are in the order $P_{70} P_{41} P_{42} P_{12}$ and $P_{70} P_{41} P_{49} P_{12}$. Take $P_{50}$ on the segment $P_{80} P_{70}$. Then take $P_{02}$ in the order $P_{12} P_{50} P_{02}$ and the line $P_{12} P_{50}$ will meet $l_{234}$ in a point $P_{52}, l_{934}$ in a point $P_{59}$, and $l_{134}$ in a point $P_{51}$, and these points are in the orders $P_{12} P_{52} P_{51} P_{50} P_{02}$ and $P_{12} P_{59} P_{51} P_{50} P_{02}$. $P_{02} P_{32}$ contains points $P_{30}$ on $l_{034}$ and $P_{61}$ on $l_{134}$, in the order $P_{02} P_{30} P_{61} P_{32}$. $P_{02} P_{42}$ meets $l_{034}$ in $P_{40}$ and $l_{134}$ in $P_{71}$, in the order $P_{02} P_{41} P_{71} P_{42}$. The line $P_{31} P_{30}$ meets $P_{52} P_{50}$ in a point $P_{01}$, in the orders $P_{31} P_{30} P_{01}$ and $P_{02} P_{01} P_{50}$. $P_{41} P_{40}$ also meets the line $P_{12} P_{02}$ in a point which by Axiom $\mathrm{X}$ is the same as $P_{01}$, from the Desargues configuration (2).

Now the line $P_{12} P_{49}$ meets $l_{134}$ in $P_{81}$, and $l_{034}$ in $P_{60}$, and these points are in the order $P_{02} P_{60} P_{51} P_{49}$. The line $P_{41} P_{60}$ meets the segment $P_{02} P_{50}$ in $P_{01}^{\prime}$. But $P_{01}^{\prime}$ can not be $P_{01}$, since $P_{40}$ and $P_{60}$ are distinct. Also $P_{41} P_{60}$ and $P_{31} P_{30}$, since each enters the triangle $P_{02} P_{30} P_{60}$ at a vertex, meet in a point $P_{x}$. But this point is not collinear with $P_{12}$ and $P_{02}$, since $P_{31} P_{30}$ and $P_{41} P_{40}$ meet $P_{12} P_{02}$ in distinct points, $P_{01}$ and $P_{01}^{\prime}$. Then we should have the Desargues configuration

* Note that the proof of the theorem as given would not be valid except for this restriction. The result is proved in general in Theorems VII and VIII. 


$$
\begin{array}{ccc} 
& Q_{34} \\
P_{30} & P_{31} & P_{32} \\
P_{60} & P_{41} & P_{49} \\
\hline P_{12} & P_{02} & P_{x}
\end{array}
$$

In this configuration, if $l_{034}, l_{134}$, and $l_{934}$ are in pencil $Q_{34}$, as we have supposed, then by Axiom $\mathrm{X}$ the points $P_{12}, P_{02}, P_{x}$ must be collinear. But we have proved that they are not collinear, and hence the lines $l_{034}, l_{134}$, and $l_{934}$ are not in pencil.

ThEOREM V. If $l_{045}, l_{145}$, and $l_{245}$ are three lines in a non-intersecting pencil, and $l_{045}, l_{145}$, and $l_{345}\left(l_{245} \neq l_{345}\right)$ are three lines in a non-intersecting pencil, then two of these lines are always between the other two.

Proof. Since no two of the lines meet, it is only necessary to find a single segment joining points of two of them, and containing points of the remaining two, as the lemma to Theorem III shows.

Let the regions into which the plane is divided by the lines $l_{045}$ and $l_{145}$ be denoted by $++;-+;--$ (cf. Theorem $g$, p. 146), the first sign referring to division by $l_{045}$ and the second to division by $l_{145}$, and the notation so used that a point in the ++ region is on the opposite side of $l_{045}$ from $l_{145}$, and a point in the - - region is on the opposite side of $l_{145}$ from $l_{045}$. The remaining points not on the lines are in -+ .

Since neither $l_{245}$ nor $l_{345}$ can meet either $l_{045}$ or $l_{145}$ each must lie wholly in some one of the regions,++-+ , or -- . We have then the following cases, with the conclusion as given:

\begin{tabular}{|c|c|c|c|c|c|c|c|}
\hline Case & $l_{245}$ in & $l_{345}$ in & \multicolumn{5}{|c|}{ Conclusion } \\
\hline 1 & $+t$ & $+t$ & See below. & & & & \\
\hline 2 & ++ & -+ & $l_{045}$ and $l_{345}$ & between & othe & & ines. \\
\hline 3 & ++ & -- & $l_{045}$ and $l_{145}$ & 6 & 66 & 6 & 66 \\
\hline 4 & -+ & $+t$ & $l_{045}$ and $l_{245}$ & 6 & “ & 6 & “ \\
\hline 5 & -+ & -+ & $l_{245}$ and $l_{345}$ & “6 & “6 & 66 & “ \\
\hline 6 & -+ & -- & $l_{145}$ and $l_{245}$ & "6 & 6 & 66 & .6 \\
\hline 7 & -- & $+t$ & $l_{145}$ and $l_{045}$ & 6 & 6 & 6 & 66 \\
\hline 8 & -- & -+ & $l_{145}$ and $l_{345}$ & 6 & “6 & 66 & 66 \\
\hline 9 & -- & -- & See below. & & & & \\
\hline
\end{tabular}

In cases 2 to 8 inclusive, the conclusion is obvious and cases 1 and 9 differ only in notation. The proof is made only for case 9. (Fig. 7.)

Take any points $P_{62}$ and $P_{73}$ on $l_{245}$ and $l_{345}$ respectively, and $P_{67}$ any point in ++ . If $P_{62}, P_{73}$ and $P_{67}$ are collinear, the theorem follows at once. If not, the segment $P_{67} P_{62}$ contains points $P_{60}$ on $l_{045}$ and $P_{61}$ on $l_{145}$. The segment 
$P_{67} P_{73}$ contains points $P_{70}$ on $l_{045}$ and $P_{71}$ on $l_{145}$. The lines $P_{60} P_{71}$ and $P_{70} P_{61}$ determine a point $P_{01}$. The points $P_{67}$ and $P_{01}$ determine a line containing points $P_{80}$ on $l_{045}$ and $P_{81}$ on $l_{145}$ in the order $P_{67} P_{80} P_{01} P_{81}$. The segment $P_{61} P_{73}$ meets the line $P_{67} P_{01}$ in a point $P_{31}$. Either $P_{71} P_{31}$ and $P_{67} P_{62}$ meet in a point $P_{63}$ or they do not. If they do meet in a point, we have the triangles $P_{60} P_{71} P_{63}$ and $P_{70} P_{61} P_{73}$ on the lines $l_{045}, l_{145}$, and $P_{73} P_{63}$ whose corresponding sides meet

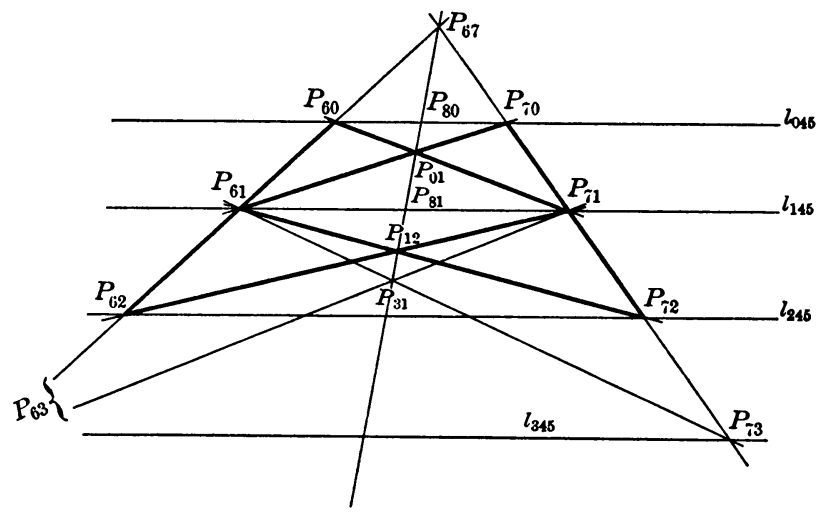

Fig. 7.

in three collinear points, $P_{67}, P_{01}, P_{31}$. Therefore $l_{045}, l_{145}$, and $P_{73} P_{63}$ are in pencil, and by Theorem IV, $P_{73} P_{63}$ is $l_{345}$ and hence $P_{63}$ is on $l_{345}$. Therefore the points $P_{60}, P_{61}, P_{62}$, and $P_{63}$ are collinear, and are in one or the other of the orders $P_{60} P_{61} P_{62} P_{63}$ or $P_{60} P_{61} P_{63} P_{62}$. In either case we have the theorem. If $P_{71} P_{31}$ and $P_{67} P_{62}$ do not meet, the line $P_{71} P_{62}$ contains a point $P_{12}$ of the line $P_{67} P_{01}$, which is in the order $P_{81} P_{12} P_{31}$; since if the points were in the order $P_{81} P_{34} P_{12}$, then $P_{71} P_{31}$ would cut the segment $P_{61} P_{62}$. Since $P_{12}$ is on the segment $P_{81} P_{31}$, it is within the triangle $P_{71} P_{61} P_{73}$, and hence the line $P_{61} P_{12}$ must cut the segment $P_{71} P_{73}$ in a point $P_{72}$. The triangles $P_{62} P_{71} P_{60}$ and $P_{72} P_{61} P_{70}$ have their corresponding sides meeting in three collinear points, $P_{67}, P_{01}$ and $P_{12}$. Therefore $P_{72} P_{62}$ is in pencil with $l_{045}$ and $l_{145}$, and by Theorem IV, $P_{72}$ is on $l_{245}$. Hence $P_{70} P_{73}$ contains $P_{71}$ on $l_{145}$ and $P_{72}$ on $l_{245}$, and hence $l_{145}$ and $l_{245}$ are between $l_{045}$ and $l_{345}$. This completes the proof.

Corollary. A triangle can always be so chosen that it will contain segments of each of the four lines $l_{045}, l_{145}, l_{245} l_{345}$ in its interior, and such that within it no two of the lines meet, and within such a triangle the order in which the four lines are cut by a transversal is invariant.

Theorem VI. If $l_{045}, l_{145}, l_{245}$ are three lines in pencil, and $l_{045}, l_{145}, l_{345}$ are three lines in pencil, then $l_{045}, l_{245}, l_{345}$ are in pencil, and $l_{145}, l_{245}, l_{345}$ are in pencil.

Proof. If $l_{045}$ and $l_{145}$ meet in a point $P_{k}$, then $l_{245}$ and $l_{345}$ pass through $P_{k}$, and we have at once the conclusion, by the corollary to Theorem II. 
If $* l_{045}$ and $l_{145}$ do not meet (Fig. 8), we will consider the interior of a triangle $T$ chosen as in the corollary to Theorem $\mathrm{V}$.

All of the points chosen below are to be taken in the interior of $T$. Let the notation $l_{x y}, l_{x}, l_{y}, l_{z}$ be so assigned to the four lines $l_{045}, l_{145}, l_{245}, l_{345}$ that if they are cut by any transversal in $T$ in the points $P_{1 c}, P_{x}, P_{y}, P_{z}$ these points

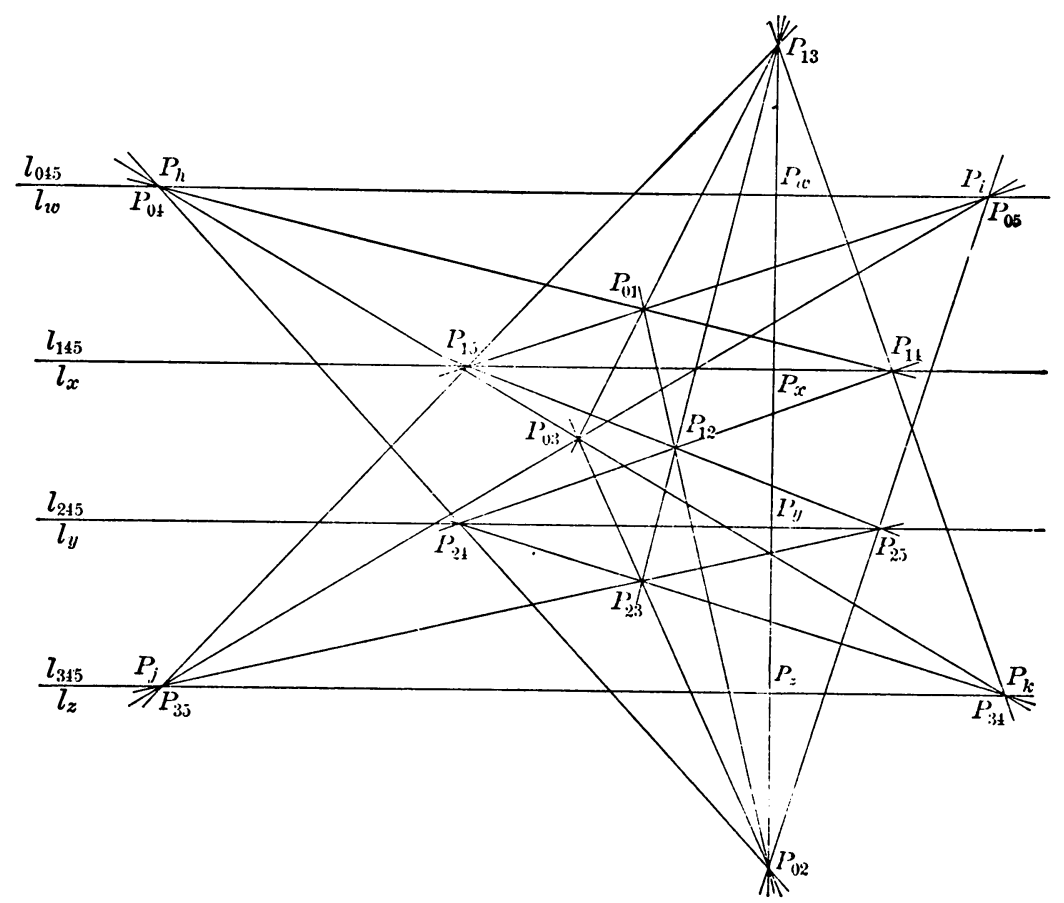

FIG. 8

will be in the order $P_{n} P_{x} P_{y} P_{z}$. Choose $P_{13}$ and $P_{02}$ in the order $P_{13} P_{x} P_{x} P_{y} P_{z} P_{02}$. Then take two points $P_{j}$ and $P_{k}$ on $l_{z}$ in the order $P_{j} P_{z} P_{k i}$, and two points $P_{h}$ and $P_{i}$ on $l_{k}$, in the order $P_{h} P{ }_{i r} P_{i}$, and such that $P_{h}$ and $P_{j}$ are on the same side of the line $P_{13} P_{12}$. The line $P_{13} P_{j}$ meets $l_{145}$ in a point $P_{15}$ and $l_{345}$ in a point $P_{35}$. The line $P_{13} P_{k}{ }_{k}$ meets the line $l_{14.5}$ in a point $P_{14}$ and $l_{315}$ in a point $P_{34}$. The line $P_{02} P_{4} P_{4}$ meets $l_{245}$ in a point $I_{24}$ and $l_{045}$ in a point $P_{014}$. The line $P_{02} I_{i}$ meets $l_{24 ;}$ in a point $I_{25}$ and $l_{04 ;}$ in a point $P_{0,5}$. Then the points $P_{14}$ and $P_{111}$ are on opposite sides of the line $P_{1,5} P_{0,5}$, hence the lines $P_{14} P_{14}$ and $P_{1: 5} P_{0,5}$ meet in a point $P_{101}$. Similarly, $P_{15} P_{25}$ and $P_{24} P_{14}$ meet in a point $P_{12} ; P_{114} P_{34}$ and $P_{0,5} P_{35}$ meet in a point $P_{43} ; P_{24} P_{34}$ and $P_{25} P_{35}$ meet in a point $P_{23}$.

* The configuration obtained in the proof of this theorem is a plane section of the three-space configuration containing six points, no three of them collinear. The configuration contains fif teen points and twenty lines. 
Then the hypothesis $l_{045}, l_{145}, l_{245}$ in pencil, and the hypothesis $l_{045}, l_{145}, l_{345}$ in pencil give respectively the Desargues configurations

$(A),(B)$

$$
\begin{array}{lllllll} 
& Q_{45} & & & Q_{45} \\
P_{04} & P_{14} & P_{24} & & P_{04} & P_{14} & P_{34} \\
P_{05} & P_{15} & P_{25} & & P_{05} & P_{15} & P_{35} \\
\hline P_{12} & P_{02} & P_{01} & & P_{13} & P_{03} & P_{01}
\end{array}
$$

Then by using the conclusions of $(A)$ and $(B)$, and remembering that any three lines through a point are in pencil, we have
$(C),(D)$

\begin{tabular}{lll} 
& $Q_{14}$ & \\
$P_{31}$ & $P_{21}$ & $P_{01}$ \\
$P_{34}$ & $P_{24}$ & $P_{04}$ \\
\hline$P_{02}$ & $P_{03}$ & $P_{23}$
\end{tabular}
\begin{tabular}{lll}
$P_{30}$ & $Q_{04}$ & $P_{10}$ \\
$P_{34}$ & $P_{24}$ & $P_{14}$ \\
\hline$P_{12}$ & $P_{13}$ & $P_{23}$
\end{tabular}

In view of $(C)$ and $(D)$ respectively, we have

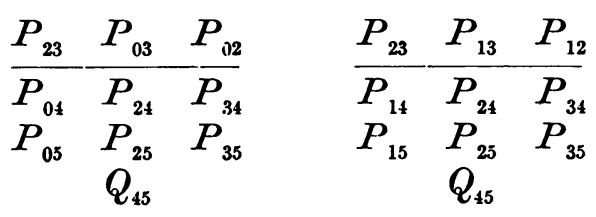

The conclusion of the theorem is expressed by $(E)$ and $(F)$ in the configurational notation.

Corollary. The set of all lines through a point constitutes a pencil which is determined by any pair of the lines.

Theorem VII. If $l_{034}$ and $l_{134}$ are two lines and $P_{32}$ is a point not on either $l_{034}$ or $l_{134}$, then there is one and only one line $l_{234}$ which passes through the point $P_{32}$ and which is in pencil with $l_{034}$ and $l_{134}$.

This is a generalization of Theorem IV, in which we have the proof for all cases except when $l_{034}$ and $l_{134}$ do not meet, and $l_{234}$ is between $l_{034}$ and $l_{134}$. We can now prove this last case and have the more general theorem by the aid of Theorem VI. We will first prove that there can not be two such lines. Suppose $l_{234}$ and $l_{534}$ are two distinct lines through $P_{34}$ each in pencil with $l_{034}$ and $l_{134}$. Then by Theorem VI, $l_{034}, l_{234}$, and $l_{534}$ are in pencil, and by Theorem I, $l_{034}, l_{134}, l_{534}$ must meet in $P_{32}$, but this contradicts the hypothesis

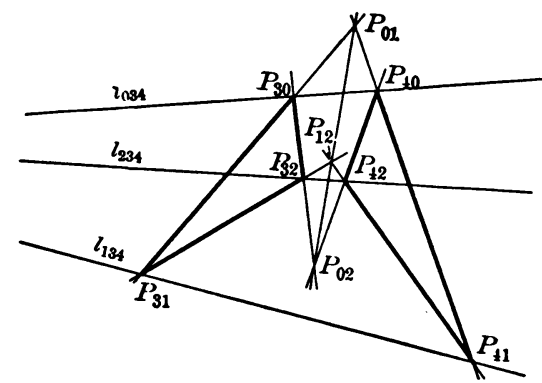

Fig. 9. that $l_{034}$ and $l_{134}$ do not meet, and hence there can not be two such lines. 
One line, and by this proof the only line $l_{234}$, through $P_{32}$ and in pencil with $l_{134}$ and $l_{134}$ will now be exhibited. (Fig. 9.) Take any point $P_{30}$ on $l_{034}$, and any point $P_{02}$ not on $l_{134}$, in the order $P_{30} P_{32} P_{02}$, and such that the segment $P_{32} P_{02}$ contains no point of $l_{134}$. Then take $P_{31}$ on the line $l_{134}$, but not on the line $P_{30} P_{32}$, and then any point $P_{01}$ in the order $P_{31} P_{30} P_{01}$. Choose $P_{40} \neq P_{30}$ on $l_{034}$ but not on the line $P_{01} P_{02}$, and any point $P_{41} \neq P_{31}$ on the line $l_{134}$, and such that these points are in the order $P_{01} P_{40} P_{41}$. Then $P_{31} P_{32}$ and $P_{01} P_{02}$ meet in a point $P_{12}$, and the segment $P_{40} P_{02}$ and the segment $P_{41} P_{12}$ meet in a point $P_{42}$. Hence we have the Desargues configuration:

\begin{tabular}{lll}
$P_{12}$ & $P_{02}$ & $P_{01}$ \\
\hline$P_{30}$ & $P_{31}$ & $P_{32}$ \\
$P_{40}$ & $P_{41}$ & $P_{42}$ \\
& $Q_{34}$ &
\end{tabular}

whence the line $P_{32} P_{42}$ is the line $l_{234}$ of the theorem.

Corollary. Any two lines determine a pencil.

In view of Theorem VII we can now speak of $P_{x} Q_{y}$, where $P_{x}$ is not the center of $Q_{y}$ as a definite line, viz., the line of the pencil $Q_{y}$ which passes through $P_{x}$. We will also speak of $Q_{x}$ as collinear with $P_{y}$ and $P_{z}$ if the line $P_{x} P_{y}$ belongs to the pencil $Q_{z}$; also if the line $P_{x} Q_{y}$ is a line of the pencil $Q_{z}, P_{x} Q_{y} Q_{z}$ will be said to be collinear. Collinearity, when used in this sense, of course, does not imply order relations. A line can belong to any number of pencils, viz., the pencils determined by it and each of the lines through some point not on it. But two pencils can not have more than one line in common, by Theorem VII. Since a point $Q_{x}$ always determines uniquely a pencil, viz., the pencil of which it is the center, we may use any point as a pencil, when we mean this pencil. Points will be so used, in speaking of ranges, as in the definition of " in range," points might have been used for the pencils, as indicating the pencils of which they are centers.

\section{SeCtion II.}

\section{Consequences of Axioms $I-X I$.}

The theorems of Section 2 will have to do with ranges and their properties. It is evident from the definitions of pencils and "in range," that any three points of a line are the centers of three pencils which are in range. The two triangles whose sides are lines of three pencils in range, and the joins of whose vertices are in pencil, will be said to be perspective triangles.

Suppose $Q_{1}, Q_{2}, Q_{3}$ are any three distinct pencils, and $T$ is any triangle not containing in its interior or on any of its sides any segment common to any two of these pencils. Let $P_{a} P_{b}^{\prime \prime} P_{c}$ be any three points in $T$ such that $P_{a} P_{b}^{\prime \prime}$ is a 
line of $Q_{1}$ and $P_{a} P_{c}$ is a line of $Q_{2}$. (Fig. 10.) Then the lines $P_{c} Q_{1}$ and $P_{b}^{\prime \prime} Q_{2}$ meet in a point $P_{1}$ of $T$, or do not. If not, let any point of $P_{c} Q_{1}$ on the same side of $P_{a} P_{c}$ that $P_{b}$ is, be called $P_{d}$; then $P_{d} Q_{2}$ will meet $P_{a} P_{b}^{\prime \prime}$ in a point $P_{b}$ which may be used for $P_{b}^{\prime \prime}$.

Such a figure $\left(P_{1} P_{b} P_{c} P_{d l}\right)$ will be called a quadrilatercl in $T$ on $Q_{1}$ and $Q_{2}$. Some one at least of the lines $P_{a} Q_{3}, P_{b} Q_{3}, P_{c} Q_{3}, P_{a} Q_{3}$ enters the quadrilateral $P_{a} P_{b} P_{c} P_{c}$, and by a proper assigmment of notation it may be considered $P_{a} Q_{3}$. Then $P_{a} Q_{3}$ will meet either the segment $P_{c} P_{d}$ or the segment $P_{b} P_{1}$ in a point $P_{, \prime}^{\prime \prime}$ or $P_{, \prime}^{\prime}$ respectively. In the first case, the line $P_{, \prime \prime}^{\prime \prime} Q_{2}$ meets the segment $P_{a} P_{b}$ in a point $P_{b}^{\prime \prime}$, and $P^{\prime \prime \prime}$ and $P_{b}^{\prime \prime}$ may be used as a new $P^{\prime}$ and $P^{\prime}{ }_{b}$, respectively. In the second case, the line $P_{, 1}^{\prime} Q_{1}$ meets the segment $P^{\prime}{ }^{\prime} P_{c}^{\prime}$ in a point $P_{c}^{\prime}$, and $P_{c}^{\prime}$ and $P_{c}^{\prime}$ may be used as $P_{c}$ and $P_{c}$ respectively, and hence the points $P_{a} P_{b} P_{c} P_{a}$ can be so chosen that they form a quadrilateral on $Q_{1}$ and $Q_{2}$, and so that $P^{\prime}{ } P_{d}$ is

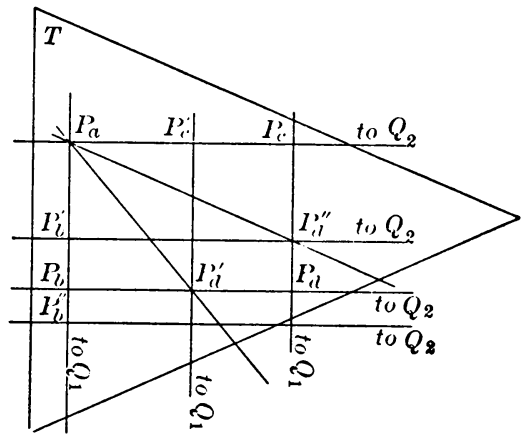

Fig. 10. a line of $Q_{3}$. Such a figure will be called a quadrilateral on $Q_{1}, Q_{2}$ and $Q_{3}$, in $T$. Any line $l_{1}$ of $Q_{1}$ and any line $l_{2}$ of $Q_{2}$, which are within the quadrilateral, meet each other in a point of the quadrilateral.

The notation for a Desargues configuration will sometimes be written, when the notation is in the form of Definition 7, D. C. (34)-(012), and when in the form of Axiom XI, D. C. (012)-(34), for shortness in writing as this may readily be expanded to the previous form.

Theorex VIII. If $Q_{01}$ and $Q_{02}$ are any two distinct pencils, and $l$ is any

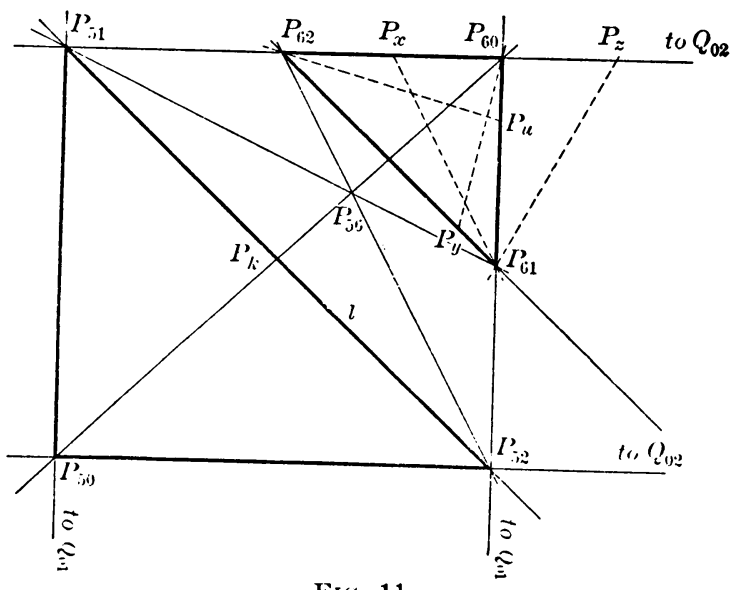

FI(i. 11. line not belonging to either $Q_{01}$ or $Q_{02}$, then $l$ is in one and only one pencil $Q_{12}$ which is in range with $Q_{111}$ and $Q_{122}$. (Fig. 11.)

Pronf: Let $T$ be any triangle containing in its interior a segment of $l$, but no segment of a line common to $Q_{01}$ and $Q_{0: 2}$. Choose $P^{\prime}{ }_{31}$ and $I^{\prime}{ }_{52}$ on $l$, in $T$, and such that $I_{51} Q_{111}$ and $I_{52} Q_{1,2}$ meet in a point $P_{\text {s. }}$ of $T$, and such that $I^{\prime}{ }_{i 11} Q_{111}$ and $I_{i 1} Q_{v 2}$ meet in a 
point $P_{60}$ of $T$. Then the segments $P_{51} P_{52}$ and $P_{60} P_{50}$ meet in a point $P_{k}$; choose $P_{56}$ in the order $P_{k} P_{56} P_{60}$. Then $P_{52} P_{56}$ meets the segment $P_{51} P_{60}$ in a point $P_{62}$, and $P_{51} P_{56}$ meets the segment $P_{52} P_{60}$ in a point $P_{61}$. Then from the triangles $P_{50} P_{51} P_{52}$ and $P_{60} P_{61} P_{62}$ perspective in $P_{56}, P_{51} P_{52}$ and $P_{61} P_{62}$ determine a pencil $Q_{12}$ which is in range with $Q_{01}$ and $Q_{02}$.

Suppose there is a pencil $Q_{12}^{\prime}$, distinct from $Q_{12}$, such that $l$ is a line of $Q_{12}^{\prime}$, and such that $Q_{01}, Q_{02}$, and $Q_{12}^{\prime}$ are in range. Then at least one of the lines $P_{60} Q_{12}^{\prime}, P_{61} Q_{12}^{\prime}, P_{62} Q_{12}^{\prime}$ must enter the triangle $P_{60} P_{61} P_{62}$, unless $P_{60} Q_{12}^{\prime}$ is identical with $P_{60} Q_{01}$ or $P_{60} Q_{112}$. But either of these was avoidable by a new choice of $P_{52}$ and $P_{51}$, since $P_{52}$ and $P_{51}$ might have been any points on the segment $P_{51} P_{52}$.

Case $I$. Suppose $P_{60} Q_{12}^{\prime}$ cuts the segment $P_{61} P_{62}$ in $P_{y}$. Then choose a point $P_{z}$ in the order $P_{51} P_{60} P_{z}$ such that $P_{z} Q_{12}^{\prime}$ passes through $P_{61}$, or a point $P_{61}^{\prime}$ on the segment $P_{61} P_{60}$. Then since $P_{56}$ was chosen arbitrarily on the segment $P_{k} P_{60}$, it may be chosen so that for some point $P_{z}$ in order $P_{51} P_{60} P_{z}, P_{z} Q_{12}^{\prime}$ passes through $P_{61}$. Then since $Q_{01} Q_{02} Q_{12}^{\prime}$ are in range, the triangles $P_{51} P_{52} P_{50}$ and $P_{61} P_{8} P_{60}$ are in perspective. But $P_{61} P_{51}$ and $P_{60} P_{50}$ meet in $P_{56}$, and $P_{52} P_{8}$ does not pass through $P_{56}$. Hence this case is impossible.

Case II. $P_{61} Q_{12}^{\prime}$ meets the segment $P_{60} P_{62}$ in a point $P_{x}$. Then since $Q_{01}$, $Q_{02}$, and $Q_{12}^{\prime}$ are in range, by hypothesis, $P_{60} P_{61} P_{x}$ and $P_{50} P_{51} P_{52}$ are in perspective. But $P_{60} P_{50}$ and $P_{61} P_{51}$ meet in $P_{56}$ and $P_{x} P_{52}$ does not pass through $P_{56}$. Hence this case is impossible.

Case III. $P_{62} Q_{12}^{\prime}$ meets the segment $P_{61} P_{62}$ in $P_{u}$. The proof in this case is identical with that of Case II, except for notation. Hence there can be no such pencil $Q_{12}^{\prime}$, and the theorem is proved.

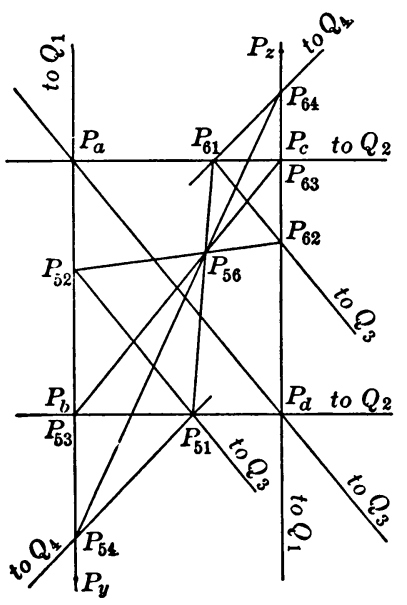

Fig. 12.

Theorem IX. If $Q_{1}, Q_{2}, Q_{3}$ and $Q_{4}$ are four distinct pencils, and $Q_{1}, Q_{2}, Q_{3}$ are in range, and $Q_{1}, Q_{2}, Q_{4}$ are in range, then also $Q_{1}, Q_{3}, Q_{4}$ are in range. (Fig. 12.)

Proof. Let $P_{a} P_{b} P_{c} P_{d}$ be a quadrilateral on $Q_{1}, Q_{2}, Q_{3}$ in a region $T$ not containing any point of a line common to any pair of the pencils $Q_{1}, Q_{2}$, $Q_{3}, Q_{4}$. Let $P_{y}$ and $P_{z}$ be any points in $T$ in the orders $P_{a} P_{b} P_{y}$ and $P_{d} P_{c} P_{z}$ respectively. Let $P_{51}$ be any point on the segment $P_{b} P_{d}$ such that $P_{51} Q_{4}$ meets the segment $P_{a} P_{y}$ in a point $P_{54}$. Let $P_{61}$ be any point on the segment $P_{a} P_{c}$, such that $P_{61} Q_{4}$ meets the segment $P_{d} P_{z}$ in a point $P_{61}$. $\quad P_{51} Q_{3}$ meets the segment $P_{a} P_{b}$ in a point $P_{52}$, and $P_{61} Q_{3}$ meets the segment $P_{c} P_{c l}$ in a point $P_{62}$. Since $P_{61}, P_{51}$ are on one pair of opposite sides of the quadrilateral, and 
$P_{62}, P_{52}$ are on the other pair of opposite sides, $P_{61} P_{51}$ and $P_{62} P_{52}$ meet in a point $P_{56}$, of the interior of the quadrilateral. If $P_{b}$ and $P_{c}$ be called $P_{53}$ and $P_{63}$ respectively, we have the Desargues configuration (123)-(56), whence $P_{53} P_{63}$ also passes through the point $P_{56}$. But from the hypothesis that $Q_{1}$, $Q_{2}$ and $Q_{4}$ are in range, and the triangles $P_{51} P_{52} P_{54}$ and $P_{61} P_{62} P_{64}$, we have the Desargues configuration (124)-(56), whence $P_{54} P_{64}$ also passes through $P_{56}$. Hence, from triangles $P_{51} P_{53} P_{54}$ and $P_{61} P_{63} P_{64}$, perspective in $P_{56}$, we have the Desargues configuration (56)-(134), whence $Q_{1}, Q_{3}$, and $Q_{4}$ are in range, and the theorem is proved.

Corollary. Any two pencils of a range determine the range.

Theorem X. If three points $P_{01}, P_{02}, P_{12}$ are in range, they are collinear. Proof. (See Fig. 13.) Let $P_{30}$ be a point not on any of the lines $P_{01} P_{02}$, $P_{01} P_{12}, P_{02} P_{12}$. Take $P_{31}$ on the line $P_{30} P_{01}$ such that the line $P_{31} P_{12}$ will

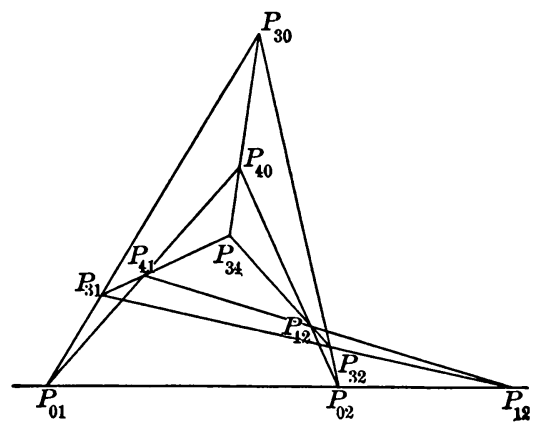

FIG. 13.

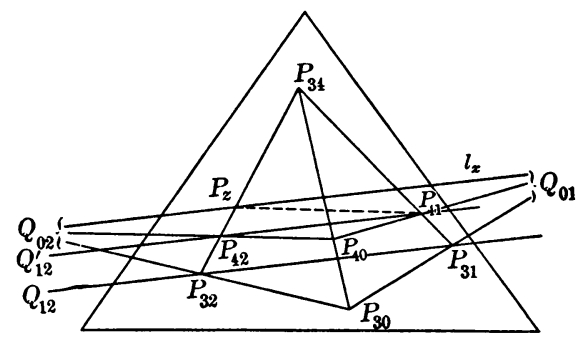

FIG. 14.

meet the segment $P_{30} P_{02}$ in a point $P_{32}$, and such that no point of any of the lines $P_{01} P_{02}, P_{01} P_{12}, P_{02} P_{12}$ is within the triangle $P_{30} P_{31} P_{32}$. Let $P_{34}$ be any point within the triangle $P_{30} P_{31} P_{32}$. Take $P_{40}$ in the order $P_{30} P_{40} P_{34}$. Then the line $P_{40} P_{01}$ will meet the segment $P_{31} P_{34}$ in a point $P_{41}$, and the line $P_{40} P_{02}$ will meet the segment $P_{34} P_{32}$ in a point $P_{42}$. But the lines $P_{42} P_{41}$ and $P_{32} P_{31}$ determine a pencil $Q_{12}^{\prime}$, which, by definition, is in range with $P_{01}$ and $P_{02}$. Hence by Theorem VIII $Q_{12}$ and $Q_{12}^{\prime}$ are identical, and we have the theorem.

TheOREM XI. If $Q_{01}, Q_{02}, Q_{12}$ are in range and $l_{x}$ is a line belonging to $Q_{01}$ and $Q_{02}$, then $l_{x}$ belongs also to $Q_{12}$. (Fig. 14.)

Proof. Let $T$ be a triangle containing in its interior a segment of the line $l_{x}$, but no center of any one of the three pencils. Let $P_{30}$ be any point in $T$ not on $l_{x}$, and $P_{31}$ a point in $T$, on line $P_{30} Q_{01}$, and $P_{32}$ a point in $I$ on $Q_{112} P_{30}$, such that $P_{31} P_{32}$ is a line of $Q_{12}$, and $P_{31}, P_{31}, P_{32}$ are all on the same side of $l_{x}$. Take $P_{34}$ on opposite side of $l_{x}$ from $P_{30}$, not on $P_{32} Q_{12}$. Suppose now $l_{x}$ is not a line of $Q_{12}$; let $P_{z}$ be the point in which $P_{32} P_{34}$ meets $l_{x}$. Then $P_{z} Q_{12}$ will meet the segment $P_{34} P_{31}$ in a point $P_{41}$, not on $l_{x}$. $P_{41} Q_{01}$ meets $P_{30} P_{34}$ in a point $P_{40}$, not on $l_{x} \cdot P_{40} Q_{02}$ meets $P_{32} P_{34}$ in a point $P_{42}$, not on $l_{x}$. Hence 
$P_{31} P_{32}$ and $P_{41} P_{42}$ determine a pencil $Q_{12}^{\prime}$ which is in range with $Q_{111}$ and $Q_{02}$. By Theorem VIII $Q_{12}^{\prime}$ is $Q_{12}$. But $P_{41} P_{z}$ was supposed to be a line of $Q_{12}$, and that would make $P_{41}$ the center of $Q_{12}$. But $Q_{12}$ does not have its center in $T$, and $P_{41}$ is in $T$, hence $l_{x}$ must be a line of $Q_{12}$.

Theonem XII. If $l_{x}$ is a line of a pencil $Q_{12}$, and $P_{02}$ and $P_{01}$ are any two distinct points of $l_{x}$ not the center of $Q_{12}$, then $Q_{12}, P_{02}$ and $P_{01}$ are in range.

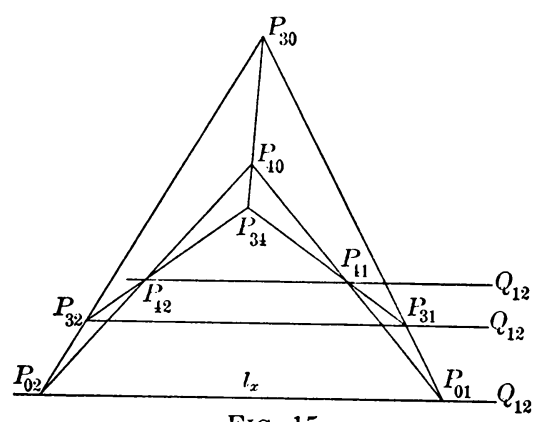

FIG. 15.

Proof. (See Fig. 15.) Let $P_{30}$ be any point not on $l_{x}$, and $P_{31}$ and $P_{32}$ two points on the same side of $l_{x}$ that $P_{30}$ is, and such that $P_{30} P_{31}$ is a line of $Q_{01}, P_{30} P_{32}$ is a line of $Q_{112}$, and $P_{31} P_{32}$ is a line of $Q_{12}$. Let $P_{3 i}$ be any point within the triangle $P_{30} P_{31} P_{32}$. Let $P_{40}$ be any point in the order $P_{30} P_{40} P_{34}$. Then $P_{40} Q_{1}$ meets the segment $P_{31} P_{34}$ in a point $P_{41}$, and $P_{40} Q_{02}$ meets the segment $P_{34} P_{32}$ in a point $P_{42}$. Then the line $P_{42} P_{41}$ belongs to the pencil $Q_{12}$, from the Desargues configuration, (043)-(12), and hence the theorem is true.

Theorem XIII. If $l_{x}$ belongs to $Q_{01}$ and $Q_{02}$, then any other pencil $Q_{12}$ to which $l_{x}$ belongs is in range with $Q_{01}$ and $Q_{02}$. Let $P_{x}$ and $P_{y}$ be two points of $l_{x}$ not centers of $Q_{01}$ or $Q_{02}$ or $Q_{12}$. Then $P_{x} P_{y} Q_{01}, P_{x} P_{y} Q_{02}, P_{x} P_{y} Q_{12}$ are in range, by Theorem XII. Then by Theorem IX, $P_{x} Q_{01} Q_{02}$ and $P_{x} Q_{01} Q_{12}$ and therefore $Q_{01}, Q_{02}, Q_{12}$ are in range.

Theorem XIV. Any two distinct ranges $r_{023}$ and $r_{123}$ have in common one and only one pencil, $Q_{23}$. (Fig. 16.)

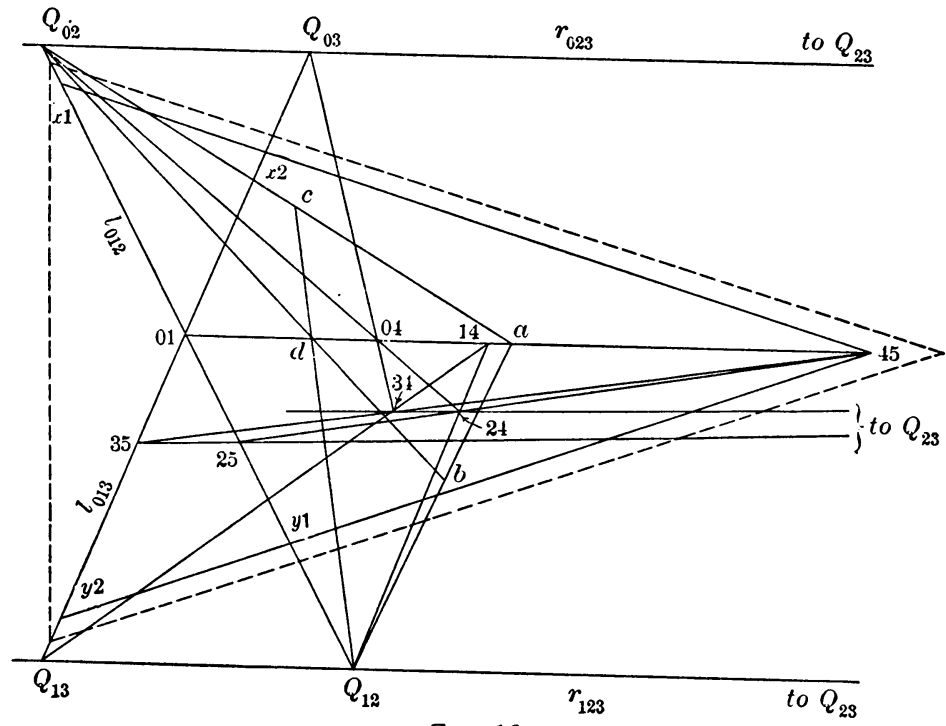

FIG. 16. 
Proof. Through any point $P_{01}$, not a point of either $r_{023}$ or $r_{123}$, draw lines $l_{013}$ and $l_{012}$, which with $r_{023}$ and $r_{123}$ determine respectively the pencils $Q_{03}$ and $Q_{13}, Q_{02}$ and $Q_{12}$. Choose $P_{45}, P_{x 2}$ and $P_{y 1}$ such that $P_{x 2}$ is on the line $l_{013}$ and $P_{y 1}$ is on the line $l_{012}$, and such that $P_{45} P_{x 2}$ meets $l_{012}$ in a point $P_{x 1}$ and $P_{45} P_{y 1}$ meets $l_{013}$ in a point $P_{y 2}$ in the orders $P_{x 1} P_{01} P_{y 1}$ and $P_{x 2} P_{01} P_{y 2}$, and such that all of these points lie within a triangle containing no points of $r_{123}$ or $r_{023}$. Take a quadrilateral $P_{a} P_{b} P_{c} P_{d}$ on $Q_{12}$ or $Q_{02}$, and $P_{01}$, and such that $P_{a}$ and $P_{d}$ are on the segment $P_{01} P_{45}$, and such that $P_{c} P_{45}$ and $P_{b} P_{45}$ are lines within the triangles $P_{45} P_{x 1} P_{01}$ and $P_{45} P_{y 2} P_{01}$ respectively. Take $P_{04}$ and $P_{14}$ on the segment $P_{a} P_{d}$, and such that $P_{04} P_{03}$ and $P_{14} Q_{13}$ meet at a point $P_{34}$ within the quadrilateral $P_{a} P_{b} P_{c} P_{a} ; P_{04} Q_{02}$ and $P_{14} Q_{12}$ meet at a point $P_{24}$ within the quadrilateral. Then $P_{45} P_{24}$ meets $l_{012}$ in a point $P_{25}$ and $P_{45} P_{34}$ meets the line $l_{013}$ in a point $P_{35}$. Then $P_{25} P_{35}$ and $P_{24} P_{34}$ determine a pencil $Q_{23}$ which is in range with $Q_{02} Q_{03}$ from D. C. (45)-(023), and in range with $Q_{12} Q_{13}$ from D. C. (45)-(123).*

That there is only one such pencil is an immediate consequence of Theorem IX. For if there were two such pencils, then these would determine any range containing them, and hence the ranges $r_{023}$ and $r_{123}$ would not be distinct.

Theorem XV. If any ten pencils $Q_{i j}(i, j=1, \cdots, 5 ; i \neq j)$ are such that no four of them are in range, and $Q_{i j} Q_{i k} Q_{j k}(i, j, k=1,2,3,4,5$; $i \neq j \neq k$, and $i, j, k \neq 1,2,3$ in any order) are in range $r_{i j k}$, then also $Q_{12}$, $Q_{13}$ and $Q_{23}$ are in range $r_{123}$, and conversely.

The theorem is the general Desargues theorem, pencils and ranges replacing points and lines, and forming the configuration $\left|\begin{array}{cc}10 & 3 \\ 3 & 10\end{array}\right|$. This configuration, for the purpose of the proof, is enlarged to a configuration $\left|{ }_{5}^{21}{ }_{35}^{3}\right|, P_{i j}$ or $Q_{i j}, l_{i j k}$ or $r_{i j k}(i, j, k=1, \cdots, 7)$, the new lines or points being within or crossing a region $T$, say the inside of a triangle, containing no point of the original ranges. The eleven points added form two perspective five-points $P_{1,2,3,4,5-6}$ and $P_{1,2,3,4,5-7}$, perspective from a point $P_{67}$.

Choose $P_{36}$, any point in $T$ (Fig. 17), and draw $P_{30} Q_{3 j}(j=1,2,4)$; in $T$, choose points $P_{16}$ and $P_{26}$, on $P_{36} Q_{13}$ and $P_{36} Q_{23}$ respectively, and such that $P_{16} Q_{14}$ and $P_{26} Q_{24}$ meet in a point $P_{46}$ of $T$, and $P_{26} Q_{25}$ and $P_{16} Q_{15}$ meet in a point $P_{56}$ of $T$. Take $P_{67}$ in $T$, but neither collinear with any two points, nor on any line yet chosen, and draw $P_{67} P_{6 j}(j=1 \ldots 5)$. Take $P_{17}$ on the segment $P_{67} P_{16}$, and draw $P_{17} Q_{12}$. This will cut the segment $P_{67} P_{26}$ in a point $P_{27}$. $P_{17} P_{14}$ meets segment $P_{67} P_{46}$ in a point $P_{47}$, which is also on the line $P_{27} Q_{24}$, from the D. C. (67)-(124). [That is, $P_{27} P_{47}$ and $P_{26} P_{46}$ determine a pencil which is in range with $Q_{14}$ and $Q_{12}$, and since $Q_{24}$ is a pencil of the line $P_{26}{ }_{26} P_{46}$, it is this pencil.] Also $P_{17} Q_{15}$ meets the segment $I_{56}^{\prime} P_{67}$ in a point $I_{57}^{\prime}$, and from

* The configuration used in the proof of this theorem is a degeverate case of the configuration $\left|\begin{array}{cc}15 & 4 \\ 3 & 20\end{array}\right|$, of notation $P_{i j}, l_{i j k}(i, j, k=0, \cdots, 5)$ in which the points $P_{01}, P_{05}$ and $P_{15}$ are coincident. 
the D. C. (67)-(125), $P_{26} P_{56}$ and $P_{27} P_{57}$ determine a pencil which is in range with $Q_{15}$ and $Q_{12}$, and is therefore $Q_{25}$. From the D. C. (67)-( $\left.i j k\right),(i=1,2$; $j, k=3,4,5, j \neq k) P_{j 6} P_{k 6}$ and $P_{j 7} P_{k 7}$ belong to $Q_{j k}$. Hence $Q_{34}, Q_{35}$,

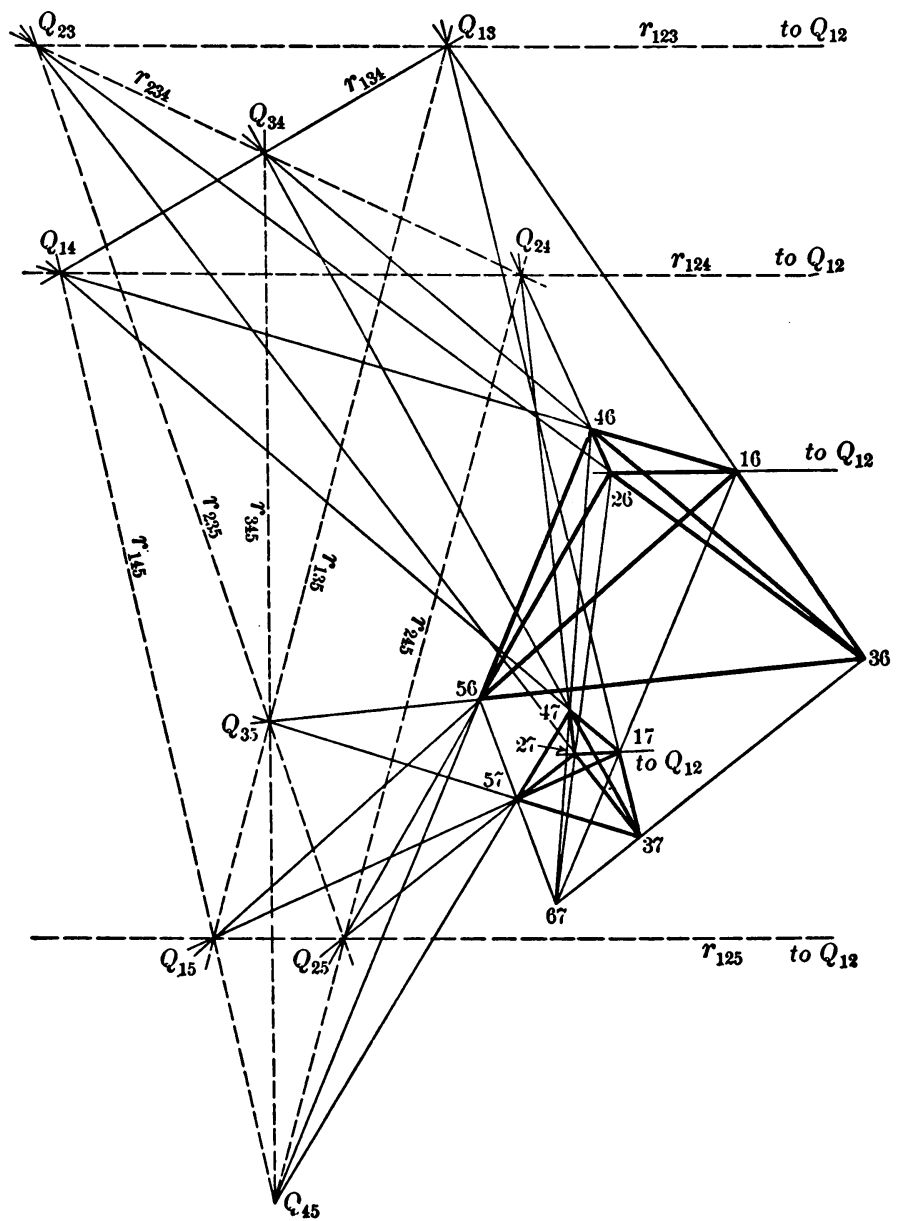

Fig. 17.

$Q_{45}$ are in range, from the D. C. (67)-(345), and we have established the first part of the theorem.

The same configuration serves for the proof of the converse theorem, if the points are chosen in a slightly different order. Choose in $T$ a point $P_{36}$, then $P_{46}$ and $P_{56}$ such that $P_{46} I_{56}^{\prime}$ is a line of $Q_{56}, P_{56} Q_{25}$ and $P_{46} Q_{24}$ meet in a point $P_{26}$ of $T$, and $P_{56} Q_{15}$ and $P_{46} Q_{14}$ meet in a point $P_{16}$ of $T$. Choose $P_{67}$ in $T$, not on any line or range of the figure so far determined. On the segment $P_{67} P_{36}$ choose $P_{37}$. Then $P_{37} Q_{35}$ meets the segment $P_{56} P_{67}$ in a point $P_{57}$. The line $P_{57} Q_{45}$ meets the segment $P_{46} P_{67}$ in a point $P_{47}$. Then from 
the D. C. (67)-(345), $P_{37} P_{47}$ is a line of $Q_{34}$. Then $Q_{14} P_{47}$ meets the segment $P_{16} P_{67}$ in a point $P_{17}$. From D. C. (67)-(145) and Theorem VIII, $P_{17} P_{57} Q_{15}$ are collinear. We have now hypotheses for the configuration (67)-(134) and (67)-(135), from which, and Theorem VIII, we have the collinearities $P_{16} P_{36} Q_{13}$, $P_{17} P_{37} Q_{13}$. The line $P_{47} Q_{24}$ meets the segment $P_{20} P_{67}$ in a point $P_{27}$, and from the D. C. (67)-(245) we have $P_{27} P_{57} Q_{25}$ collinear. From the D. C.'s (67)-(234) and (67)-(235) we have the collinearities $P_{26} P_{36} Q_{23}$ and $P_{27} P_{37} Q_{23}$.

But from the D. C.'s (67)-(123), (67)-(124), (67)-(125), the lines $P_{16} P_{26}$ and $P_{17} P_{27}$ determine a pencil $Q_{12}$ which is in range with $Q_{13} Q_{23}, Q_{14} Q_{24}$, $Q_{15} Q_{25}$ respectively, and this proves the theorem.

\section{SeCrion III.}

\section{Generalization of Order.}

In this section it is shown that if some one range of pencils is omitted from the set of all pencils, the remaining subset of pencils has the property, that, with a suitable definition of order, its elements satisfy the Axioms I-XI, a well-known property of the elements of a projective plane.

Before giving the definition of order it will be useful to prove certain lemmas.

Lемma I. If $Q_{h}, Q_{i}, Q_{j}, Q_{k}$ are any four distinct pencils of a range $r_{h i j k}$, and $T_{x} \equiv P_{x h i} P_{x h j} P_{x i j}$ is a triangle whose sides belong to $Q_{h}, Q_{i}, Q_{j}$ respectively (i. e., the line $P_{x h i} P_{x h j}$ belongs to the pencil $Q_{h}$, etc.), all of wbose vertices are on the same side of $r_{h i j k}$, then of the three statements

1) $P_{x h i} Q_{k}$ cuts the segment $P_{x j h} P_{x i j}$ in a point $P_{x j k}$;

2) $P_{x h j} Q_{k}$ cuts the segment $P_{x h i} P_{x i j}$ in a point $P_{x i k}$;

3) $P_{x i j} Q_{k}$ cuts the segment $P_{x l i} P_{x h j}$ in a point $P_{x h k}$;

one and only one is true.

Proof. Since the vertices of the triangle are all on one side of the range $r_{l i j k}$, no side of the triangle is $r_{l i j k}$. Then the lines $P_{x h i} Q_{k}, P_{x h j} Q_{k}, P_{x i j} Q_{k}$ are all distinct lines of a pencil, whose center, if it exists, is not within the triangle. If the pencil has a center, the lemma follows at once from theorem $g$ of the introduction. If it has no center, it follows from Theorem III. It is evident that by a proper assignment of the notation $Q_{h}, Q_{i}, Q_{j}$ the true statement may be made any desired one of the three.

Lemma II. If $T_{3}$ and $T_{4}$ are two triangles satisfying the hypothesis of Lemma I, and such that in the triangle $T_{3}$, the line $P_{3 h j} Q_{k}$ meets the segment $P_{3 h i} P_{3 i j}$ in a point $P_{3 i k}$, then also the line $P_{4 h j} Q_{k}$ meets the segment $P_{4 h i} P_{4 i j}$ in a point $P_{4 i k}$.

Proof. Case I. (Fig. 18.) When no pair of corresponding sides of the triangles $T_{3}$ and $T_{4}$ lie in the same line, and no vertices are in common. The lines 
$P_{3 h i} P_{4 l i}, P_{3 h j} P_{4 h j}, P_{3 i j} P_{4 i j}$ are in pencil $Q_{34}$. The line $P_{3 i k} Q_{34}$ meets the seg. ment $P_{4 h i} P_{4 i j}$ in a point $P_{4 i k}$, by theorems on division of a plane by lines and Theorem I, if $Q_{34}$ has a center, and by Theorem IV, if $Q_{34}$ has no center. But

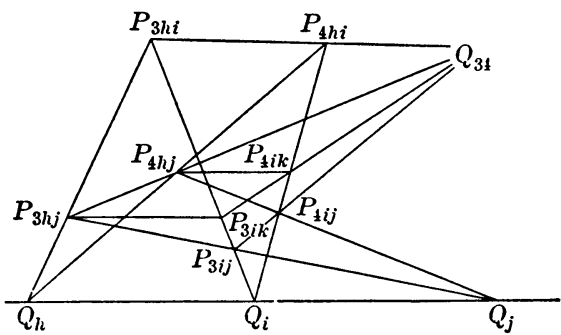

FIG. 18.

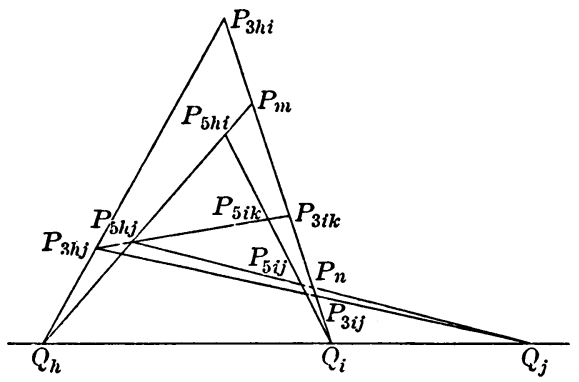

FIg. 19.

$P_{4 h j} P_{4 i k}$ and $P_{3 h j} P_{3 i k}$ determine a pencil in range with $Q_{h}$ and $Q_{i}$, and hence $Q_{k}$.

Case II. (Fig. 19.) In case some parts of the triangles $T_{3}$ and $T_{4}$ are coincident, we can make the proof as follows. It is always possible to obtain a triangle $T_{5}$, satisfying hypothesis of Lemma II, all of whose parts are distinct from all the parts of $T_{3}$ and $T_{4}$, and such that $P_{5 h j} Q_{k}$ meets the segment $P_{5 h i} P_{5 i j}$ in a point $P_{5 i k}$, whence the theorem follows by Case I. Such a triangle $T_{5}$ is obtained as follows. Within the triangle $T_{3}$ and on the segment $P_{3 h j} P_{3 i k}$ but on no side of $T_{4}$, choose a point $P_{5 h j}$. The line $P_{5 h j} Q_{h}$ will meet the segment $P_{3 h i} P_{3 i k}$ in a point $P_{m}^{\prime}$, and the line $P_{5 h j} Q_{j}$ will meet the segment $P_{3 i k} P_{3 i j}$ in a point $P_{n}$. Choose a point $P_{5 l i}$ on the segment $P_{5 h j} P_{m}$ and not on any line of $T_{4}$. Then $P_{5 h i} Q_{i}$ will meet the lines $P_{5 h j} P_{n}$ and $P_{5 h j} P_{3 i k}$ in points $P_{5 i j}$ and $P_{5 i k}$ respectively, and the triangle $P_{5 h j} P_{5 h i} P_{5 i j}$ is the triangle required.

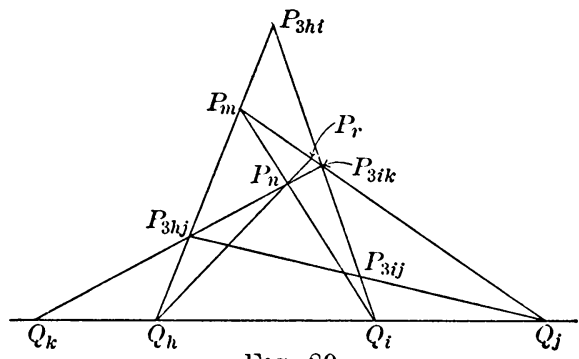

Lemma III. (Fig. 20.) If $Q_{h}, Q_{i}$, $Q_{j}, Q_{k}$ are four distinct pencils of a range, $r_{h i j k}$, and $T_{3}$ is a triangle satisfying the hypotheses of Lemma II, then in any triangle

FIG. 20 .

$$
T_{x} \equiv\left\{\begin{array}{l}
P_{x h i} P_{x h k} P_{x i k} \\
P_{x h j} P_{x h k} P_{x j k} \\
P_{x i j} P_{x i k} P_{x j k}
\end{array}\right\}
$$

all of whose vertices are on the same side of $r_{h i j k}$, and whose sides belong to the pencils

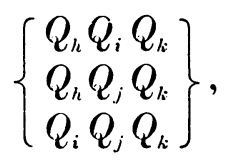


respectively, the line and segment

meet in a point

$$
\left\{\begin{array}{c}
P_{x i k} Q_{j} \\
P_{x h j} Q_{i} \\
P_{x i k} Q_{h}
\end{array}\right\}, \quad\left\{\begin{array}{l}
P_{x h i} P_{x h k} \\
P_{x h l k} P_{x j k} \\
P_{x i j} P_{x j k}
\end{array}\right\},
$$

$$
\left\{\begin{array}{l}
P_{x h j} \\
P_{x k i} \\
P_{x j h}
\end{array}\right\} \text {. }
$$

Proof. The line $Q_{j} P_{3 i k}$ can not meet the segment $P_{3 h j} P_{3 i j}$, hence meets the segment $P_{3 h j} P_{3 h i}$ in a point $P_{m}$, and hence from the triangle $P_{3 h i} P_{3 h j} P_{3 i k}$ and Lemma II we have the first part of the lemma. Similarly, $P_{m} Q_{i}$ must meet the segment $P_{3 h j} P_{3 i k}$ in a point $P_{n}$, and hence from the triangle $P_{m} P_{3 h j} P_{3 i k}$ and Lemma II we have the second part of the lemma. Also $P_{n} Q_{h}$ must meet the segment $P_{m} P_{3 i k}$ in a point $P_{r}$, and from the triangle $P_{m} P_{n} P_{3 i k}$ and Lemma II we have the third part of the lemma.

The range of pencils omitted from the set $Q_{k}$ will be called $r_{\infty}$, and pencils not on $r_{\infty}$ will be denoted by the notation $S_{k}$.

Definition of ORder. (Fig. 21.) Three pencils have order relations if and only if they are distinct, lie in a range, and no one of them lies on $r_{\infty}$. Three such pencils $S_{i}, S_{j}, S$, of a range $r_{i j k}$ meeting $r_{\infty}$ in $Q_{\infty}$, are in the order $S_{i} S_{j} S_{k}$ if and only if in a triangle $P_{x i j} P_{x i k} P_{x j k}$, whose sides belong to the pencils $S_{i}, S_{j}, S_{k}$ respectively, and all of whose vertices are on the same side of $r_{\infty}$ and $r_{i j k}$, the line $P_{x i k} Q_{\infty}$ meets the segment $P_{x i j} P_{x j k}$ in a point $P_{j \infty}$.

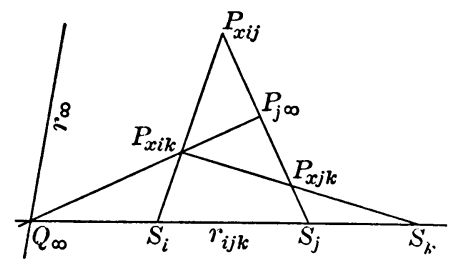

Fia. 21.

It will now be shown that the set of pencils $S$, subject to the above definition of order, satisfies the Axioms I-XI.

Axiom $I$ is evident, since every point is the center of a pencil.

Axiom II is evident from the symmetry of the definition of order.

Axiom III follows from Lemma I.

Axiom IV is evident from definition.

Axiom $\mathrm{V}$ is evident from definition and Lemma III.

Axiom VI is a consequence of Theorem IX.

Axion VII is obvious.

Axioms IX, X, XI are evident, since all points are centers of pencils, and they hold for the set of all pencils.

Axıом VIII. (Fig. 22.) This will be paraphrased as follows. If in the set $S_{k}, S_{145}, S_{245}$, and $S_{345}$ are any three pencils not in range, and $S_{235}$ is a pencil in the order $S_{245} S_{345} S_{235}$, and $S_{135}$ is a pencil in the order $S_{145} S_{135} S_{345}$, 
then there exists a pencil $S_{125}$ which is in the order $S_{145} S_{125} S_{245}$, and which is in range with $S_{135}$ and $S_{235}$.

Proof. The ranges $S_{135} S_{235}$ and $S_{145} S_{245}$ are distinct, and hence they determine a pencil $Q_{125}$. Select a triangle $T$ containing in its interior no point of any of the ranges $S_{245} S_{125}, S_{245} S_{345}, S_{145} S_{345}, r_{\infty}$. All points selected below are to be taken within the triangle $T$. Call the pencils common to $r_{\infty}$ and $S_{145} S_{245}$,

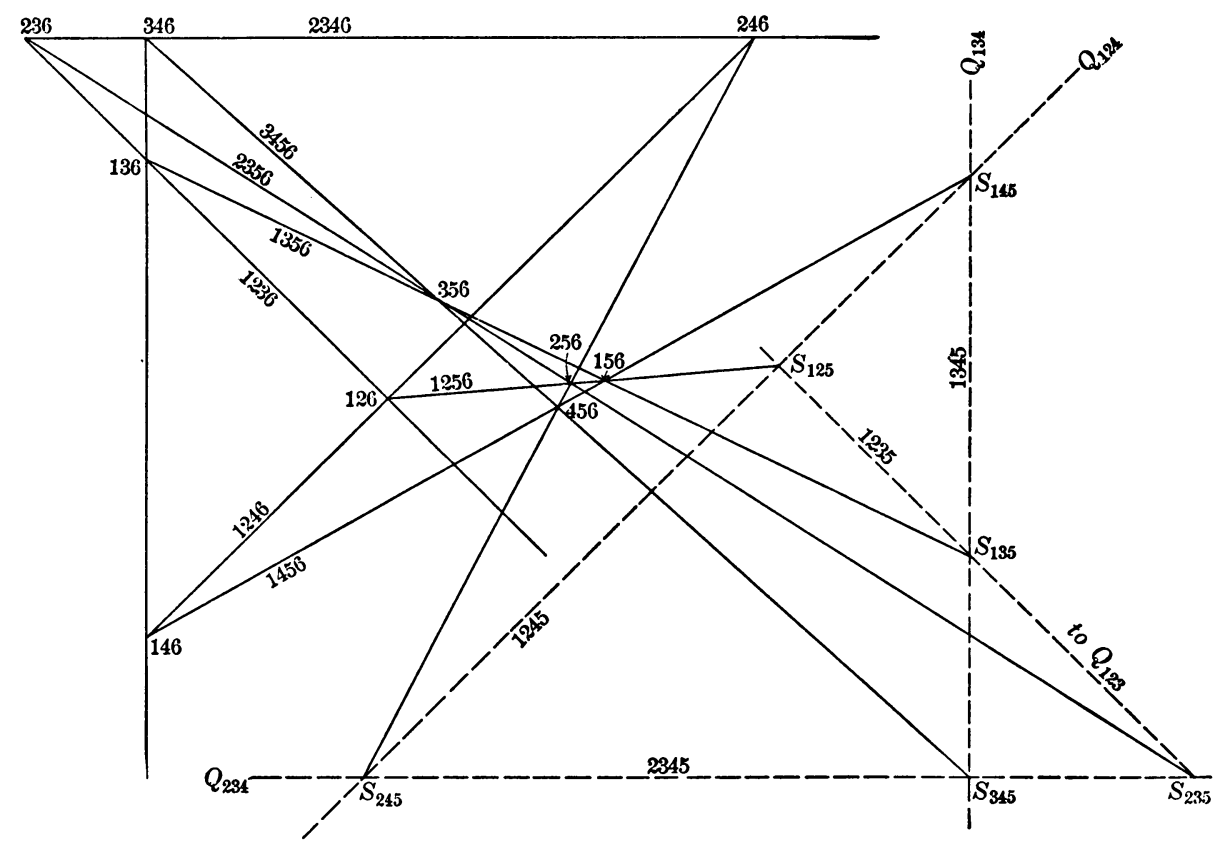

FIG. 22.

$S_{245} S_{345}, S_{145} S_{345}, S_{135} S_{235}$, respectively $Q_{124}, Q_{234}, Q_{134}, Q_{123}$. Choose in $T$ a point $P_{456}$, and then points $P_{146}, P_{246}, P_{346}$ on the lines $P_{456} S_{145}, P_{456} S_{245}$, $P_{456} S_{345}$, respectively, and such that the lines $P_{346} P_{246}, P_{346} P_{146}$, and $P_{146} P_{246}$. belong to the pencils $Q_{234}, Q_{134}$, and $Q_{124}$, respectively. From the order $S_{245} S_{345} S_{235}$ of the hypothesis, $P_{346} S_{235}$ meets the segment $P_{456} P_{246}$ of the triangle $P_{246} P_{346} P_{546}$. Hence it is possible to find in $T$ points $P_{236} P_{356} P_{256}$ in the orders $P_{236} P_{346} P_{246}, P_{346} P_{356} P_{456}, P_{246} P_{256} P_{456}, P_{236} P_{356} P_{256}$. (Equally well might another set of orders have been secured.)

Then $P_{356} S_{135}$ meets $P_{456} S_{145}$ in a point $P_{156}$ of $T$ or does not. If it does, $P_{156}$ is in the order $P_{146} P_{456} P_{156}$, from the order $S_{145} S_{135} S_{345}$ of the hypothesis and the triangle $P_{346} P_{146} P_{456}$. If not, choose in $T$ a point $P_{156}^{\prime}$ in the order $P_{146} P_{456} P_{156}^{\prime}$, and from the order $S_{145} S_{135} S_{345}$ of the hypothesis, and the triangle $P_{346} P_{146} P_{456}$, by Lemmas I, III, $P_{456} S_{135}$ meets the segment $P_{346} P_{146}$, and $P_{156}^{\prime} S_{135}$ will meet the segment $P_{456} P_{356}$ in a point $P_{356}^{\prime}$. The line 
$P_{356}^{\prime} S_{235}$ will meet the segment $P_{456} P_{256}$ in $P_{256}^{\prime}$, and will or will not meet $P_{346} P_{246}$ in a point $P_{x}$ of $T$. Take $P_{236}^{\prime}$ in the order $P_{256}^{\prime} P_{356}^{\prime} P_{236}^{\prime}$, and in the order $P_{356}^{\prime} P_{236}^{\prime} P_{x}$ if $P_{x}$ exists, and such that the line $P_{236}^{\prime} Q_{234}$ meets the segment $P_{346} P_{146}$ in a point $P_{346}^{\prime}$, and the segment $P_{146} P_{246}$ in $P_{246}^{\prime}$. In this case use the points marked with primes in the further discussion, where points without the primes are used. The line $P_{356} S_{135}$ will meet the segment $P_{346} P_{146}$ in a point $P_{136}$. The sets of three pencils, $P_{256} P_{356} P_{456}$ and $S_{215} S_{315} S_{415}$ have corresponding ranges belonging to the pencils $Q_{345}, Q_{245}$, and $Q_{235}$ of the range $r_{2355}$, and the lines $S_{456} S_{145}$ and $P_{356} S_{135}$ meet in $P_{156}$. Hence the line $P_{256} S_{125}$ also passes through the point $P_{156}$ (by Theorem XIII), and from the order $P_{456} P_{356} P_{346}$, the point $P_{136}$ is in the orders $P_{156} P_{356} P_{136}$ and $P_{346} P_{136} P_{146}$. Also, the line $P_{236} P_{136}$ meets the line $P_{146} P_{246}$ in a point $P_{126}$ in the orders $P_{236} P_{136} P_{126}$ and $P_{146} P_{126} P_{246}$. From the sets of three pencils $P_{136} P_{236} P_{436}$ and $S_{135} S_{235} S_{435}$ "perspective" in $P_{356}$, the line $P_{136} P_{236}$ belongs to the pencil $Q_{123}$. From the sets of three pencils $S_{125} S_{325} S_{425}$ and $P_{126} P_{326} P_{426}$, whose sides belong in pairs to the three pencils $Q_{124}, Q_{234}, Q_{123}$, respectively, $P_{126}$ is on the line $P_{256} P_{156}$. From the triangle $P_{156} P_{256} P_{456}$, the line $P_{456} Q_{124}$ cuts the segment $P_{156} P_{256}$, since it is not either side line of the triangle, and since it can not meet the segment $P_{146} P_{246}$. Hence if $Q_{125}$ is not on $r_{\infty}$, it is $S_{125}$ and in the order $S_{145} S_{125} S_{245}$. That it is not on $r_{\infty}$ may be seen as follows: $S_{245} S_{145}$ meets $r_{\text {oc }}$ in $Q_{124}$, of which $P_{146} P_{246}$ is a line. If this line were the same as $P_{126} P_{256}, P_{156}$ would be on the line $P_{146} P_{246}$, contrary to the hypothesis. Hence the axiom holds.

The configuration used in the proof may be indicated schematically by $\left|\begin{array}{cc}20 & 4 \\ 3 & 15\end{array}\right|$, as may readily be verified from the figure.

\section{Section IV.}

In terms of the plane set of pencils $S$, already exhibited in Section III, a new set of elements will now be defined which will be shown to form a three dimensional space, as mentioned in the introduction. Upon the assumption of a plane satisfying the ordinary incidence and order relations, and, furthermore, the parallel axiom, Hilbert* demonstrates that the validity of the Desargues theorem is the necessary and sufficient condition that the plane constitutes a part of a threedimensional geometry with analogous relations. That the Desargues theorem holds for a plane of such a three space is well known. On the other hand, by the aid of an algebra of segments based upon the Desargues theorem and the parallel axiom, and a resulting analytic geometry, Hilbert exhibited an analytic three-space containing the original plane, while ScHOR $\nmid$ later exhibited a geo-

* Grundlagen der Geometrie, Festschrift, \& 29.

† Schоr, D., Neuer Beweis eines Satzes aus den Grundlagen der Geometrie von Hilbert. Mathematische Annalen, vol. 58 (1904), pp. 427-433. 
metric three-space without the algebra of segments. The method here used is analogous to that of Schor, but makes no use of the parallel axiom; this is possible in view of the use of the ideal elements already introduced on the basis of the Desargues theorem. The ideal elements of the resulting three-space, which were not considered by either Hilbert or Schor, are also developed in terms of the original plane system.

Let three special distinct pencils $Q_{v}, Q_{v}, Q_{10}$ of $r_{\infty}$ be chosen. Ranges $S_{k}$ distinct from $r_{\infty}$ and belonging to $Q_{u}, Q_{v}, Q_{10}$ respectively, will be denoted by $u_{k}, v_{k}, w_{k}$, respectively. A 3-point will then be defined as follows:

Every triple of ranges $u_{k}, v_{k}, w_{l k}$, not in pencil, is a 3-point. Also since every pencil $S_{k}$, with the pencils $Q_{u}, Q_{v}, Q_{w}$, respectively, determines three ranges $u_{k}, v_{k}, w_{k}$, respectively, which are in pencil, and conversely every three ranges $u_{l k}, v_{k}, w_{l k}$ which are in pencil determine a pencil $S_{k}$, every pencil $S_{k}$ will also be called a 3-point, and the notation $T_{k} \equiv u_{k}, v_{k}, w_{k}$ will be used for 3-points of either class. If $T_{x}$ is a 3-point, $u_{x}$ and $v_{x}, u_{x}$ and $w_{x}, v_{x}$ and $w_{x}$, determine three pencils $W_{x}, V_{x}$ and $U_{x}$, respectively, which are coincident or distinct according as $T_{k}$ is or is not a pencil.

Definition. Three 3-points $T_{x}, T_{y}, T_{z}$ are in the order $T_{x} T_{y} T_{z}$ if and only if the pencils $U_{x}, U_{y}, U_{z}$ are in the order $U_{x} U_{y} U_{z}$ as pencils.

In view of the Lemma to Theorem III, the order $U_{x} U_{y y} U_{z}$ implies also the orders $V_{x} V_{y} V_{z}$ and $W_{x} W_{y} W_{z}$, and conversely. Also all three, or any one, of the three 3-points having order relations may be pencils, but if two are pencils the third is a pencil.

If three 3-points $T_{x}, T_{y}, T_{z}$ are collinear, $U_{x}, U_{y}, U_{z}$ are in range, $V_{x}, V_{y}, V_{z}$ are in range, $W_{x}, W_{y}, W_{z}$ are in range, and these three ranges are in pencil $Q_{x y z}$.

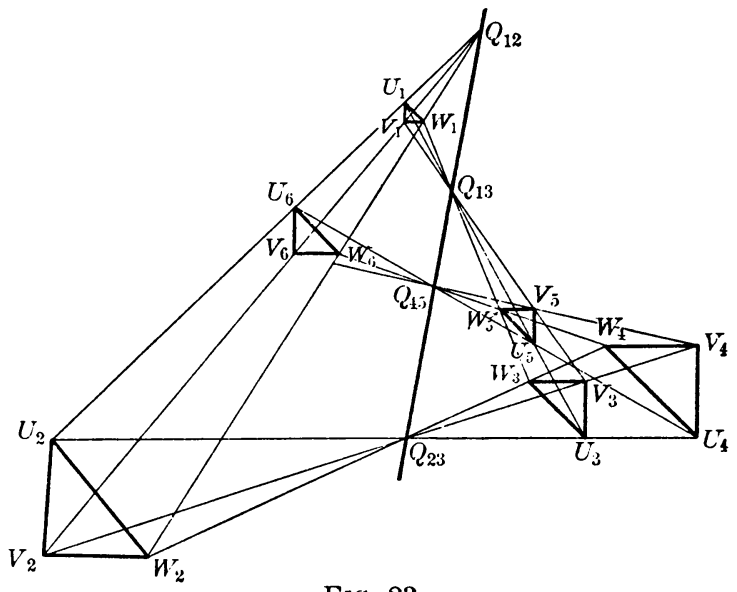

FIG. 23.

It will now be shown that the set of elements $T_{k}$ satisfies the Axioms I-XI, except IX, and in place of IX possesses a property IX', characteristic of threedimensionality.

The validity of Axioms IVII inclusive follows immediately from our definitions and their validity with respect to the elements $S$.

Axion VIII. (Fig. 23.) General C'ase. If $T_{1}, T_{2}, T_{3}$ are any three non-collinear 3 points, forming a triangle, and $T_{4}$ is a 3-point in the order $T_{2} T_{3} T_{4}$, and $T_{5}$ is a 3 -point in the order $T_{3} T_{5} T_{1}$, then there exists a 3-point $T_{6}$ in the order $T_{1} T_{6 j} T_{2}$, and on the line $T_{4} T_{5}$. 
Proof. Let $Q_{12}$ be the pencil determined by the ranges $U_{1} U_{2}, V_{1} V_{2}, W_{1} W_{2}$; $Q_{45}$ the pencil determined by the ranges $U_{4} U_{5}, V_{4} V_{5}, W_{4} W_{5} ; Q_{23}$ the pencil determined by $U_{2} U_{3}, V_{2} V_{3}, W_{2} W_{3}$; and $Q_{13}$ the pencil determined by the ranges $U_{1} U_{3}, V_{1} V_{3}, W_{1} W_{3}$.

Then we have two Desargues configurations.

$$
\begin{array}{ccccccc} 
& (A) & & & (B) \\
& Q_{10} & & & Q_{10} \\
V_{1} & V_{2} & V_{3} & & U_{3} & U_{4} & U_{5} \\
U_{1} & U_{2} & U_{3} & & V_{3} & V_{4} & V_{5} \\
\hline Q_{23} & Q_{13} & Q_{12} & & Q_{45} & Q_{13} & Q_{23}
\end{array}
$$

Hence from $(A)$ and $(B)$ together we have $Q_{23}, Q_{13}, Q_{12}, Q_{45}$ in range, and this gives us the hypotheses of the Desargues configuration $(C),(D),(E)$.
\begin{tabular}{lll} 
& $(C)$ & \\
& $Q_{23}$ & \\
$Q_{45}$ & $W_{4}$ & $U_{4}$ \\
$Q_{12}$ & $W_{2}$ & $U_{2}$ \\
\hline$Q_{0}$ & $U_{6}$ & $W_{6}$
\end{tabular}
\begin{tabular}{lll} 
& $(D)$ & \\
& $Q_{23}$ & \\
$Q_{45}$ & $W_{4}$ & $V_{4}$ \\
$Q_{12}$ & $W_{2}$ & $V_{2}$ \\
\hline$Q_{u}$ & $V_{6}$ & $W_{6}$
\end{tabular}
$Q_{23}$

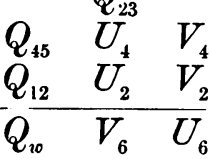

From $(C),(D)$, and $(E)$, we know that the set of ranges $U_{6} W_{6}, U_{6} V_{6}$, $V_{6} W_{6}$ is a 3-point. It still remains to show that this 3-point is in the order $T_{1} T_{6} T_{2}$. But the figure formed by the pencils $Q_{n}, Q_{v}, Q_{10}, U_{1}, U_{2}, U_{3}, U_{4}$, $U_{5}, U_{6}$ is precisely the figure of Axiom VIII, Section III, whence $U_{6}$ is in the order $U_{1} U_{6} U_{2}$, and hence $T_{6}$ is in the order $T_{1} T_{6} T_{2}$. This also shows that no one of the three pencils $U_{6}, V_{6}, W_{6}$ could be on the range $r_{\infty}$.

It is obvious that one or more of the 3-points $T_{1}, T_{2}, T_{3}, T_{4}, T_{5}, T_{6}$ could be pencils, but the above general proof is easily modified to fit all the special cases, and the details of these proofs are omitted.

Axion IX is evidently not satisfied.

Axıом $\mathrm{X}$. The hypothesis of Axiom $\mathrm{X}$ gives at once

$$
\begin{array}{lll}
X_{12} & X_{02} & X_{01} \\
\hline X_{40} & X_{41} & X_{42} \\
X_{30} & X_{31} & X_{32} \\
& X_{34}^{*} &
\end{array} \quad(X=U, V, W),
$$

the asterisk indicating that $U_{34}^{*}, V_{34}^{*}, W_{34}^{*}$ may or may not be a 3-point, but simply that $U_{34}^{*}, V_{34}^{*}, W_{34}^{*}$ exist as pencils, whether or not on $r_{\infty}$.

We have then at once the configurations,

$$
\begin{aligned}
& \begin{array}{lll} 
& Q_{z} & \\
X_{m 4} & X_{m 3} & X_{m n} \\
Y_{m 4} & Y_{m 3} & Y_{m n} \\
\hline Q_{m n 3} & Q_{n n 4} & Q_{m 34}
\end{array} \\
& \begin{array}{ccc} 
& Q_{z} & \\
X_{n 4} & X_{n 3} & X_{n m} \\
Y_{n 4} & Y_{n 3} & Y_{n m} \\
\hline Q_{n m 3} & Q_{n m 4} & Q_{n 34}
\end{array}
\end{aligned}
$$


for $z, X, Y=u, V, W ; v, W, U ; w, U, V$; and $m, n=1,2 ; 0,1 ; 0,2$.

From these configurations we have at once the collinearities $Q_{124} Q_{134} Q_{123} Q_{234}$, $Q_{013} Q_{014} Q_{034} Q_{134}$ and $Q_{023} Q_{024} Q_{034} Q_{234}$. These give the hypotheses for the configurations

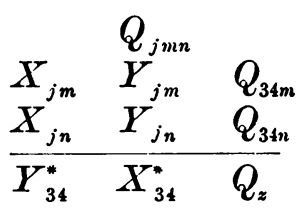

for $j=3,4 ; m, n=0,1 ; 0,2 ; 1,2$; and $X, Y, z=U, V, w ; V, W, u$; $W, U, v$.

From this it follows that $U_{34}^{*}, V_{34}^{*}, W_{34}^{*}$ is a 3-point if these pencils do not lie on $r_{\infty}$, and if it is not a 3-point they do lie on $r_{\infty}$.

Now let $T_{31}, T_{41} ; T_{30}, T_{40} ; T_{32}, T_{42}$ be any pairs whatever of 3-points on the same 3-lines, such that $T_{01}, T_{02}, T_{12}$ exist, and it must be shown that $T_{01}, T_{02}, T_{12}$ are collinear.

We have at once the Desargues configurations

$$
\begin{array}{ccc}
X_{31} & X_{34}^{*} & \\
X_{41} & X_{40} & X_{32} \\
\hline X_{02} & X_{12} & X_{01}
\end{array}
$$

and then the configurations

$$
\begin{array}{lll} 
& Q_{z} & \\
X_{i k} & X_{i l} & X_{i j} \\
Y_{i k} & Y_{i l} & Y_{i j} \\
\hline Q_{i j l} & Q_{i j k} & Q_{i k l}
\end{array}
$$

where $i, j, k, l=0,1,2,3,4$ but all different and $z, X, Y=u, V, W$; $v, W, U ; w, U, V$. These give the collinearities $Q_{i j k}, Q_{i j l}, Q_{i k l}, Q_{j k l}$ $(i, j, k, l=0,1,2,3,4$ but all different).

These give the hypotheses for the configurations

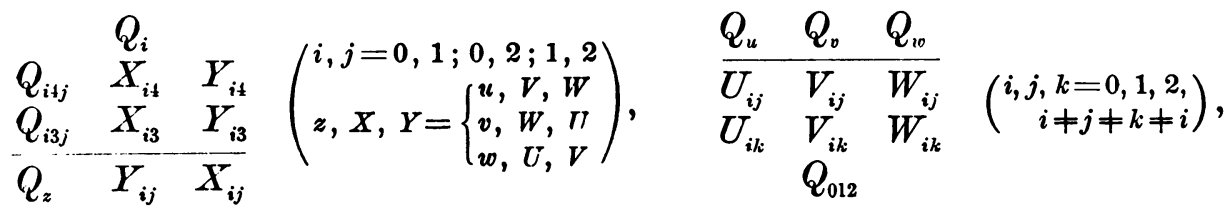

whence $T_{01}, T_{02}$, and $T_{12}$ are collinear, and we have the conclusion of the theorem.

Axiom XI. The hypothesis of three 3-points in a range implies the existence of a pair of triangles the joins of whose corresponding vertices are in a pencil. 
But, conversely, by Axiom X, this implies that the three 3-points are in a 3line. But any pair of triangles with sides through these three collinear points are in pencil, by definition. Hence Axiom XI holds.

Definition. A tetrahedron is a set of four planes, determined by four noncoplanar points taken in triples. The existence of such points follows from the fact that Axiom IX does not hold.

Definition. A 3-space is the set of all points collinear with two distinct points of a tetrahedron.

Theorem. (Fig. 24.) The set of 3-points $T$ constitutes a 3-space.

Proof. Let $T_{3} \equiv U_{3}, V_{3}, W_{3}$ (distinct) be any 3-point. Then, since $U_{3}$, $V_{3}, W_{3}$ as pencils of the set $S$, are themselves 3-points, we have a tetrahedron, $T_{3}, U_{3}, V_{3}, W_{3}$. Let $T_{4} \equiv U_{4}, V_{4}, W_{4}$ be any other 3-point. Then $U_{3} U_{4}$, $V_{3} V_{4}, W_{3} W_{4}$, belong to a pencil $Q_{34}$. If $Q_{34}$ is not a pencil of the range $r_{\infty}$, it is a 3-point of the plane determined by $U_{3}, V_{3}, W_{3}$, and $T_{4}$ is collinear with $T_{3}$ and $Q_{34}$, and hence is a point of the 3-space $T_{3} U_{3} V_{3} W_{3}$.

If $Q_{34}$ is on $r_{\infty}$, choose a new pencil $V_{3}^{\prime}$ in the order $V_{3} V_{3}^{\prime} U_{3}$. Then $V_{3}^{\prime} Q_{u}$ meets $U_{3} Q_{v}$ in $W_{3}^{\prime}$, and we have a 3-point $T_{3}^{\prime} . \quad T_{3}^{\prime}$ is in the plane determined by $V_{3} U_{3} T_{3}$, and $T_{3}^{\prime}$ and $T_{4}$ belong to a pencil $Q_{34}^{\prime} \neq Q_{34}$, hence

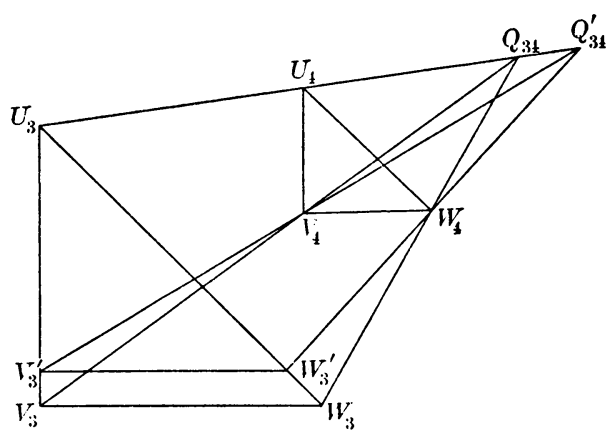

FIG. 24. not on $r_{\infty}$. Hence $T_{4}$ is collinear with two distinct points of the tetrahedron, and the points $T$ constitute a 3 -space.

Ideal elements of the 3-space $T$. We have now a three-dimensional set of points satisfying order relations, and the ideal 3-space elements might be introduced in it directly, by the methods of Bonola, Schur, or Veblen.* It is interesting, from the point of view of the present paper, to do this in terms of the original plane elements.

Let $T_{3} \equiv U_{3}, V_{3}, W_{3}$ and $T_{4} \equiv U_{4}, V_{4}, W_{4}$ be any two 3-points. The ranges $U_{3} U_{4}, V_{3} V_{4}, W_{3} W_{4}$ meet the range $r_{\infty}$ in three pencils $Q_{n^{\prime}}, Q_{0^{\prime}}, Q_{w^{\prime}}$, which are in general distinct. The set of three pencils $Q_{n^{\prime}}, Q_{v^{\prime}}, Q_{r^{\prime}}$, whether or not distinct, will be defined as an ideal element of the 3 -space $T$, and will be said to lie on the 3 -line $\left(U_{3} L_{4}^{r}, V_{3} V_{4}, W_{3} W_{4}\right)$. If these ideal 3-points are adjoined to the set $T$, giving a set $T^{\prime}$, the points of $T^{\prime}$ will not satisfy the order relations as defined, since the set $Q$ did not, but will, evidently, have the following three properties :

Any two planes determine one and only one line.

${ }^{*} \mathrm{Cf}$. the previous citations.

Trans. Am. Math. Soc. 12 
Any two lines of a plane determine a point.

The general Desargues theorem.

In short, the set of elements $T^{\prime}$ has all the intersectional properties of a projective 3-space, except those which are dependent on Pascal's theorem. These must be excepted, since Pascal's theorem is independent of the Desargues theorem (cf. Hilbert).

\section{Section V. \\ Extension to $n$-Space.}

The method of Section IV may now be extended to cover the definition of $n$-space in terms of the plane set of elements already considered. We choose on the line $r_{\infty}$ of the set of elements $Q, n$ pencils, $Q_{1}, Q_{2}, \ldots, Q_{n}$. Any set of $n$ ranges $r_{i}(i=1,2, \ldots, n)$, one belonging to each of the pencils $Q_{1}, \ldots, Q_{n}$, respectively, and each distinct from the range $r_{\infty}$, will be defined as an $n$-point, and denoted by the notation $N_{x}$. These $n$ ranges, taken in pairs, determine $n(n-1) / 2$ pencils (not necessarily distinct), $S_{x, i j}(i, j=1, \ldots, n ; i \neq j)$. Let two such n-points be $N_{x}$ and $N_{y}$. The pencils $S_{x, i j}$ and $S_{y, i j}$ $(i, j=1, \ldots, n ; i \neq j)$ determine a range $r_{x y, i j}$ such that $r_{x y, i j}, r_{x y, i k}, r_{x y, j k}$ $(i, j, k=1, \cdots, n ; i \neq j \neq k \neq i)$ are in pencil.

The set of $\frac{1}{2} n(n-1)$ ranges $r_{x y, i j}$ will be called an $n$-line. An $n$-point $N_{z}$ will be said to be on the $n$-line $n_{x y}$, if and only if, the pencil $S_{z, i j}$ belongs to the range $r_{r y, i j}(i, j=1, \cdots, n ; i \neq j)$. Its order will be said to be the same as the order of the pencils $S_{x, i j}, S_{y, i j}, S_{z, i j}$. That this definition of order is unique is easily seen as follows. Each $i, j, k$ may be thought of as fixing a 3 -space geometry in which the order for $i j, i k, j k$ is unique, and by a sequential process the result holds for all of the ranges, and hence we have the general result for the $n$-space. Hence we may write the order $N_{x} N_{y} N_{z}$ as implying the orders $S_{r, i j} S_{y, i j} S_{z, i j}$ for each triple of values for $i, j, k$, and conversely. The n-point $N_{x}$ will be said to be the same as the pencil $S_{x}$ if $S_{x, i j}$ is $S_{x}^{y}$ for every $i j$, since $S_{x}$ will in this case completely determine the $n$-point.

It can now be shown that this $n$-point geometry satisfies all the axioms except that of closure. Axioms I-VII inclusive follow immediately from the definitions. The validity of Axiom VIII may be shown as follows. The axiom states that $N_{1}, N_{2}, N_{3}$ are the vertices of a triangle, and if $N_{4}$ and $N_{5}$ are in the orders $N_{2} N_{3} N_{1}$ and $N_{1} N_{5} N_{3}$ respectively, then there exists an $n$-point $N_{6}$ in the order $N_{1} N_{t ;} N_{2}$ and on the line $N_{4} N_{5}$. Consider any triple of the pencils $Q_{1}, \cdots, Q_{n}$, of $r_{s}$, say $Q_{i}, Q_{j}, Q_{k}$. Then we have the existence of the three ranges $r_{i, i}, r_{b, j}, r_{b, k}$, of the $n$-point, $N_{6}$, from the corresponding theorem of the 3 -space theory. Similarly, if $i, j, k$ be allowed to take each set of values (distinct and of the set $1 \cdots n$ ), we obtain all of the ranges of the set forming the $n$-point $N_{t i}$, and each of the pencils $S_{t, i j}$ will be in the order required from the corresponding 3 -space theorem. Hence Axiom VIII is valid. 
That Axiom IX does not hold is evident, since each pencil $S_{x}$ is an $n$-point, but there are other $n$-points which do not lie in the plane of the pencils $Q$.

Axioms $\mathrm{X}$ and XI may be easily shown to hold by considering the pencils $Q_{1}, \ldots, Q_{n}$ of the range $r_{\infty}$ in sets of triples, and then applying the theorem already proved for each of the resulting 3 -spaces, thus obtaining the theorem for $n$-space.

That the space thus obtained is actually an $n$-space may be shown as follows : By the same method of proof as in Section IV, if for $Q_{1}, \ldots, Q_{n}$, we have an $n$-space, then by increasing the number of pencils by adding $Q_{n+1}$ to the set, we introduce new elements into the geometry. But these may all be seen to be collinear with two points of $n+1 n$-spaces, and hence constitute an $(n+1)$ space. But the theorem is true for 3-space, and hence by induction, for any value of $n$.

The ideal elements which must be adjoined to this $n$-space to make it projective are the pencils ( $n$ in number, but not necessarily distinct) belonging to the range $r_{\infty}$, in which any other $n$-line (i. e., its component ranges) meet this range $r_{\infty}$. If we call each such set an ideal $n$-point, and adjoin all such ideal points to the set already obtained, we have a set possessing the properties that any two $n$-points determine an $n$-line, any two $n$-lines determine an $n$-point, and the general Desargues Theorem. The first two of these statements are evident, and the last may readily be seen by considering the parts of the figure separately, as in the proof of Axiom VIII.

Cornell University, ITHACA, N. Y. 